Check for updates

Cite this: Phys. Chem. Chem. Phys., 2021, 23, 536

Received 24th September 2020, Accepted 2nd December 2020

DOI: $10.1039 / \mathrm{d} 0 \mathrm{cp} 05056 \mathrm{c}$

rsc.li/pccp

\section{Photoacoustic studies of energy transfer from ozone photoproducts to bath gases following Chappuis band photoexcitation $\dagger$}

\author{
Michael I. Cotterell, (D)*a Kate Szpek, (D) ${ }^{\mathrm{b}}$ David A. Tiddeman, ${ }^{\mathrm{b}} \mathrm{Jim}$ M. Haywood ${ }^{\mathrm{bc}}$ \\ and Justin M. Langridge ${ }^{b}$
}

Photoacoustic spectroscopy (PAS) is a sensitive technique for the detection of trace gases and aerosols and measurements of their absorption coefficients. The accuracy of such measurements is often governed by the fidelity of the PAS instrument calibration. Gas samples laden with $\mathrm{O}_{3}$ of a known or independently measured absorption coefficient are a convenient and commonplace route to calibration of PAS instruments operating at visible wavelengths $(\lambda)$, yet the accuracy of such calibrations remains unclear. Importantly, the photoacoustic detection of $\mathrm{O}_{3}$ in the Chappuis band $(\lambda \sim 400-700 \mathrm{~nm}$ ) depends strongly on the timescales for energy transfer from the nascent photoproducts $O\left({ }^{3} P\right)$ and $\mathrm{O}_{2}(X, v>0)$ to translational motion of bath gas species. Significant uncertainties remain concerning the dependence of these timescales on both the sample pressure and the bath gas composition. Here, we demonstrate accurate characterisation of microphone response function dependencies on pressure using a speaker transducer to excite resonant acoustic modes of our photoacoustic cells. These corrections enable measurements of photoacoustic response amplitudes (also referred to as PAS sensitivities) and phase shifts with variation in static pressure and bath gas composition, at discrete visible wavelengths spanning the Chappuis band. We develop and fit a photochemical relaxation model to these measurements to retrieve the associated variations in the aforementioned relaxation timescales for $\mathrm{O}\left({ }^{3} \mathrm{P}\right)$ and $\mathrm{O}_{2}(\mathrm{X}, v>0)$. These timescales enable a full assessment of the accuracy of PAS calibrations using $\mathrm{O}_{3}$-laden gas samples, dependent on the sample pressure, bath gas composition and PAS laser modulation frequency.

\section{Introduction}

Photoacoustic spectroscopy (PAS) has become a central technique for sensitive and accurate measurements of trace gases, ${ }^{1-6}$ and aerosol light absorption. ${ }^{7-14} \mathrm{~A}$ central and motivating feature of our research is the application of PAS to measurements of absorption coefficients $\left(\alpha_{\text {abs }}\right)$ at visible wavelengths for atmospheric aerosol, and developing PAS instruments that operate from the UK BAe-146 atmospheric research aircraft. ${ }^{15-19}$ The magnitude of aerosol light absorption, and thus the representation of absorbing aerosols in climate models, is highly uncertain. ${ }^{20,21}$ PAS is also an invaluable tool in the study of aerosol mixing state, with our spectroscopic

\footnotetext{
${ }^{a}$ School of Chemistry, University of Bristol, Bristol, BS8 1TS, UK.

E-mail: m.cotterell@bristol.ac.uk

${ }^{b}$ Observation Based Research, Met Office, Exeter, EX1 3PB, UK

${ }^{c}$ College for Engineering, Mathematics and Physical Sciences, University of Exeter, Exeter, EX4 4QF, UK

$\dagger$ Electronic supplementary information (ESI) available. See DOI: 10.1039/ d0cp05056c
}

measurements enabling changes in particle composition to be inferred and the validity of optical mixing rules (effective medium approximations) to be challenged..$^{10,22}$ Underpinning measurements of $\alpha_{\text {abs }}$ using PAS is the accurate calibration of PAS instruments. This work assesses rigorously the conditions under which PAS instruments operating at visible wavelengths are calibrated accurately using $\mathrm{O}_{3}$-laden gas, a common calibrant of photoacoustic spectrometers.

Previous work suggested that $\mathrm{O}_{3}$-laden gas samples could be a poor calibrant of PAS instruments operating at visible wavelengths. ${ }^{8}$ Meanwhile, our own PAS studies at visible wavelengths have demonstrated that calibrations using $\mathrm{O}_{3}$-laden gas can provide calibrations of high accuracy under specific conditions, ${ }^{10}$ and that the level of accuracy is sensitive to the bath gas composition. ${ }^{23,24}$ Importantly, $\mathrm{O}_{3}$ undergoes photolysis at visible wavelengths (Chappuis band) and the bath gas composition governs the timescales on which energy from the nascent photoproducts transfers into translational motion of the bath species that drives the formation of a photoacoustic pressure wave. 
One remaining knowledge gap, particularly relevant to PAS measurements from research aircraft at reduced atmospheric pressures, is the dependence of the calibration accuracy on pressure. These measurements are particularly challenging as they require methods to correct for other pressure dependencies in the photoacoustic transduction process, including the response function for the detection microphone. Moreover, direct measurements have not been reported for the photoproduct relaxation timescales that govern the accuracy of calibrations. Here, we report measurements of the variation in photoacoustic calibration coefficient (referred to as the sensitivity) with the bath gas composition (consisting of controlled fractions of $\mathrm{O}_{2}$ and $\mathrm{N}_{2}$ ) and the sample pressure over the range 300-1000 $\mathrm{hPa}$. To enable these measurements, we validate a pressure correction scheme that accounts for changes in the microphone response function and the resonance characteristics of a photoacoustic resonator that amplifies generated photoacoustic pressure waves. Importantly, we also measure the photoacoustic phase shift with respect to the periodic modulation cycle of the excitation laser source, providing a direct measurement of the relaxation timescale for the nascent $\mathrm{O}_{3}$ photoproducts. We then demonstrate closure between our measured PAS sensitivities and phase shifts with a photochemical model that describes the relaxation of Chappuis band photoproducts in different bath gas mixtures and sample pressures.

In the remainder of this introduction, Section 1.1 describes pertinent aspects to photoacoustic signal generation and detection, Section 1.2 compares approaches to PAS instrument calibration, Section 1.3 describes the impact of Chappuisband photochemistry on calibrations using $\mathrm{O}_{3}$-laden gas, and Section 1.4 outlines the remaining knowledge gaps that preclude assessments of the fidelity of $\mathrm{O}_{3}$-based calibrations that form the motivation for this work.

\subsection{Photoacoustic signal generation and detection}

A typical PAS instrument uses laser light to irradiate a light absorbing sample contained within a photoacoustic cell. ${ }^{25}$ In our detection scheme, the laser intensity $I$ is modulated periodically at an angular frequency $\omega$ such that $I(\vec{r}, t)=$ $I_{0} g(\vec{r})\left(1+\mathrm{e}^{i \omega t}\right) / 2$, in which $I_{0}$ is the intensity amplitude, $g(\vec{r})$ is the intensity distribution function with position $\vec{r}$, and $t$ is time. The sample irradiated by the laser will absorb light, and energy is deposited in the photoacoustic cell with an energy density $H(\vec{r}, t)$ described by:

$$
H(\vec{r}, t)=\alpha_{\mathrm{abs}}(\vec{r}) I(\vec{r}, t)
$$

in which $\alpha_{\text {abs }}(\vec{r})$ is the spatial distribution in the sample absorption coefficient. Following light absorption, the analyte (i.e. an aerosol particle or a gaseous absorber) is photoexcited; for aerosols, this photoexcitation energy rapidly transfers into translational energy on a rapid picosecond timescale that heats the droplet. The generation of a photoacoustic signal requires this highly localised energy to transfer to translational modes of the surrounding bath gas. The increase in bath gas translational energy close to the heated or photoexcited sample causes adiabatic expansion of the bath gas and creates a pressure disturbance. Thus, the transient nature to $H(\vec{r}, t)$ through periodic modulation of the laser intensity forms pressure (acoustic) waves in the bath gas. For coherent generation of a photoacoustic signal, we require efficient (fast) energy transfer from the sample into bath gas translational energy. Energy transfer can be slow for large aerosol particles (larger than $\sim 2 \mu \mathrm{m}$ in diameter) or those containing volatile components that facilitate energy dissipation through latent heat flux, leading to notable suppression in the generated acoustic pressure waves. $^{26-28}$ For photoexcitation of gaseous light absorbers, energy transfer to bath gas translational modes occurs through vibration-to-translation ( $\mathrm{V}-\mathrm{T}$ ) transitions and is often in competition with processes that do not contribute to acoustic signal generation. ${ }^{6,24,29}$ Such processes include photodissociation, fluorescence and vibration-to-vibration transitions. We discuss these aspects of quenching timescales and competing energy transfer pathways for photoexcited $\mathrm{O}_{3}$ in more detail below. Following their generation, acoustic pressure waves are coupled into a resonant mode of a photoacoustic cell and are amplified. This cell has an eigenmode pressure distribution that is intrinsic to the cell geometry, with an associated resonance frequency (eigenfrequency) at which the eigenmode is optimally excited. ${ }^{25,30,31}$ By modulating the laser intensity at the cell eigenfrequency, the generated photoacoustic waves resonantly excite the cell eigenmode. The quality $(Q)$ factor (which describes the acoustic energy stored in the eigenmode relative to that lost through damping processes) is typically in the range 50-90; higher $Q$ denotes larger acoustic amplification factors. A sensitive microphone is placed at an antinode in the eigenmode pressure distribution for detection of the amplified pressure response. For efficient excitation of a single pressure eigenmode $p_{n}$ (with $n$ denoting the mode index) at the corresponding eigenfrequency $f_{n}=\omega_{n} /(2 \pi)$, the acoustic pressure at the microphone location is described by: ${ }^{25,30,31}$

$$
p\left(\vec{r}_{\mathrm{M}}, \omega, t\right)=\frac{Q_{n}(\sigma-1) I_{0}}{\omega_{n}} \frac{\int g(\vec{r}) \alpha_{\mathrm{abs}}(\vec{r}) p_{n}(\vec{r}) \mathrm{d} V}{V} p_{n}\left(\vec{r}_{\mathrm{M}}\right) \mathrm{e}^{i \omega t}
$$

in which $\sigma$ is the adiabatic coefficient of the bath gas, $V$ is the cell volume, $p_{n}(\vec{r})$ is the eigenmode pressure distribution and $p_{n}\left(\vec{r}_{\mathrm{M}}\right)$ is the eigenmode pressure at the location of the microphone. The voltage output from the microphone is thus a sinusoidal waveform, the amplitude of which is referred to as the photoacoustic signal amplitude $S$. A further quantity extracted from the microphone waveform is the phase shift in the microphone response with respect to the laser power modulation $(\theta)$. This phase is a direct measure of the timescale $(\tau)$ of energy transfer to bath gas translational modes: ${ }^{6}$

$$
\theta=\tan ^{-1}\left(\omega_{n} \tau\right)
$$

In the limit of efficient energy transfer to bath gas translational modes, $S$ is directly proportional to $\alpha_{\text {abs }}$, with the constant of proportionality determined by calibration of the photoacoustic spectrometer. This calibration often governs the accuracy of absorption coefficient measurements. We now discuss strategies for calibrating PAS instruments operating at visible wavelengths. 


\subsection{Calibration of photoacoustic spectrometers}

Assuming that energy transfer to bath gas translational modes is fast and efficient, the PAS sensitivity $(C)$ is defined by:

$$
C=\frac{S}{\alpha_{\mathrm{abs}}}
$$

It should be noted that this definition of PAS sensitivity is not synonymous with the common definition as the minimum detectable signal. Researchers have used a variety of analytes for calibrating PAS instruments at visible wavelengths, including aerosols or gaseous calibrants. Aerosol calibrations commonly use laboratory-generated dry non-volatile aerosols of a known refractive index and use size classification methods to select sub-micrometre diameter aerosols, while a condensation particle counter draws aerosol through the PAS instrument and records the particle number concentration. ${ }^{8,10,22,32}$ From the controlled particle size and measured number concentration, Lorenz-Mie theory can be used to predict $\alpha_{\mathrm{abs}}$ under the assumption that the generated particles are spherical and optically homogenous. However, high uncertainties in the particle number concentration and size distribution often degrade the accuracy of these calibrations. ${ }^{22,33,34}$

In contrast, PAS calibrations using a gaseous light absorber typically pass the gas sample through both a PAS instrument and a cavity ring-down spectrometer in a serial flow configuration, with cavity ring-down spectroscopy providing an independent and direct (calibration-free) measurement of $\alpha_{\text {abs }}$ for gases. Commonly, calibration gases for visible wavelength PAS instruments contain concentrations of either $\mathrm{NO}_{2},{ }^{35-37}$ or $\mathrm{O}_{3} \cdot{ }^{10,38-40} \mathrm{NO}_{2}$ has a strong and broad absorption band across the visible spectrum. ${ }^{41}$ However, the high photolysis quantum yield for $\mathrm{NO}_{2}$ at visible wavelengths below $420 \mathrm{~nm}$ degrades the calibration accuracy. ${ }^{36,42}$ When using $\mathrm{NO}_{2}$ to calibrate PAS instruments operating at wavelengths $(\lambda)$ of 375 and $405 \mathrm{~nm}$, Nakayama et al. observed biases in $C$ of $48 \%$ and $36 \%$ respectively attributed to $\mathrm{NO}_{2}$ photolysis, while no detectable biases were observed for longer wavelength PAS instruments $(\lambda=532,781 \mathrm{~nm}) .{ }^{36}$

Ozone also has a broad absorption band (Chappuis band) over the visible wavelength range $400-700 \mathrm{~nm} .{ }^{41,43}$ Chappuis band absorption is up to five orders of magnitude weaker than that for $\mathrm{NO}_{2}$ at short visible wavelengths. Nonetheless, $\mathrm{O}_{3}$-laden gas has been used successfully to calibrate PAS instruments at discrete visible wavelengths spanning the Chappuis band. ${ }^{10,22,32,38-40}$ Until recently, the impacts of $\mathrm{O}_{3}$ photolysis in the Chappuis band on the accuracy of PAS calibrations had not been explored. However, Fischer and $\mathrm{Smith}^{23}$ and ourselves $^{24}$ have reported the strong impact of bath gas and wavelength-dependent $\mathrm{O}_{3}$ photolysis on the accuracy of these calibrations and we now discuss these dependencies.

\subsection{The impact of $\mathrm{O}_{3}$ photochemistry on calibrations of photoacoustic spectrometers}

We have previously reported accurate calibrations of our PAS instruments $(\lambda=405,514$ and $658 \mathrm{~nm})$ using $\mathrm{O}_{3}$-laden air, with $\mathrm{O}_{3}$ concentrations up to $680 \mathrm{ppmv}$ diluted into a bath gas of air. ${ }^{10,22}$ Using our $\mathrm{O}_{3}$-calibrated PAS, we compared measured aerosol absorption cross sections with those calculated by a Lorenz-Mie routine for laboratory-generated dry nigrosin aerosols. Importantly, there were no detectable biases in $C$ within the measurement uncertainty of the aerosol cross sections at the three discrete PAS wavelengths.

Recognising that researchers often use different bath gas compositions for their $\mathrm{O}_{3}$-laden calibration gases, Fischer and Smith measured the dependence of $C$ on the bath gas composition. ${ }^{23}$ The authors varied the relative bath gas fractions of $\mathrm{O}_{2}$ and $\mathrm{N}_{2}$ and recorded the associated variations in $C$ for $\lambda=532,662,780 \mathrm{~nm}$. They reported that $C$ increased with the $\mathrm{O}_{2}$ fraction and approached an asymptote in the limit of pure $\mathrm{O}_{2}$ that agreed with calibrations performed using either $\mathrm{NO}_{2}$-laden gas or using aerosol calibrants. Meanwhile, $C$ reduced by a factor of two in the limit of a pure $\mathrm{N}_{2}$ bath gas. Within measurement uncertainty, the asymptote for $C$ was attained for a bath gas $\mathrm{O}_{2}$ mass fraction $x_{\mathrm{O}_{2}} \geq 0.2$. This latter observation agrees with our aforementioned finding that accurate calibrations of PAS instruments can be achieved with $\mathrm{O}_{3}$-laden air $\left(x_{\mathrm{O}_{2}} \sim 0.25\right)$. Fischer and Smith accounted for the measured asymptotic behaviour using a kinetic model that treated the relative rates with which photoexcited $\mathrm{O}_{3}$ is quenched by either $\mathrm{O}_{2}$ or $\mathrm{N}_{2}$ bath species. Their model attributed the drop in $C$ at low $\mathrm{O}_{2}$ concentrations to inefficient energy transfer from photoexcited $\mathrm{O}_{3}$ to $\mathrm{N}_{2}$ translational modes and the authors recommended that calibrations used a bath gas of $\mathrm{O}_{2}$.

Fischer and Smith did not assess the bath gas dependence in $C$ for short visible wavelengths, such as for $\lambda=405 \mathrm{~nm}$ that is used in our instruments. Moreover, the quenching model developed by Fisher and Smith attributed the photoacoustic signal generation to direct relaxation of $\mathrm{O}_{3}^{*}$ (with the superscript * implying that $\mathrm{O}_{3}$ is in an electronically excited state) and did not account for the known photodissociation of $\mathrm{O}_{3}$ within the Chappuis band. Thus, our more recent publication assessed the bath gas composition dependence to $C$ for our own spectrometers and observed wavelength dependence to this bath gas dependency. ${ }^{24}$ Our $\lambda=658 \mathrm{~nm}$ measurements demonstrated an asymptote in agreement with Fischer and Smith's observations, while measurements for $\lambda=405 \mathrm{~nm}$ found that $C$ was maximised for $x_{\mathrm{O}_{2}}$ in the range $0.2-0.3$ and decreased by $11 \%$ as the bath gas composition tended to pure $\mathrm{O}_{2}$. We then developed a photochemical model of the dependence of $C$ on both $x_{\mathrm{O}_{2}}$ and wavelength that considered fully Chappuis band photochemistry, and the best fit of this model reproduced our observed trends in $C$. We now describe this photochemical model, that is central to this paper.

Chappuis band photoexcitation leads to prompt photodissociation to form $\mathrm{O}\left({ }^{3} \mathrm{P}\right)$ and $\mathrm{O}_{2}\left(\mathrm{X}^{3} \Sigma_{\mathrm{g}}^{-}, v>0\right)$ in which the nascent $\mathrm{O}_{2}$ photofragment is vibrationally excited: ${ }^{43}$

$$
\mathrm{O}_{3}+h \nu(\lambda=400-700 \mathrm{~nm}) \rightarrow \mathrm{O}\left({ }^{3} \mathrm{P}\right)+\mathrm{O}_{2}\left(\mathrm{X}^{3} \Sigma_{\mathrm{g}}^{-}, v>0\right)
$$

This photodissociation occurs on a picosecond timescale and can be considered instantaneous compared to our PAS 
modulation timescales (i.e. the period for the periodic laser intensity modulation) that are in the range 620-660 $\mu$ s. The subsequent photoacoustic response depends on the timescale for energy transfer $(\tau)$ from the nascent $\mathrm{O}\left({ }^{3} \mathrm{P}\right)$ and $\mathrm{O}_{2}\left(\mathrm{X}^{3} \Sigma_{\mathrm{g}}^{-}, v>0\right)$ to translational modes of bath gas $\mathrm{N}_{2}$ and $\mathrm{O}_{2}$ :

$$
C=\frac{C_{0}}{\sqrt{1+\left(\omega_{n} \tau\right)^{2}}}
$$

in which $C_{0}$ is the photoacoustic response in the limit of instantaneous energy transfer from the analyte to the bath gas. ${ }^{6,44}$ Eqn (5) shows that $C$ is maximised when $\tau$ is much less than the photoacoustic modulation timescale $2 \pi / \omega_{n}$. We then write contributions to $C$ arising from energy transfer from the separate $\mathrm{O}\left({ }^{3} \mathrm{P}\right)$ and $\mathrm{O}_{2}\left(\mathrm{X}^{3} \Sigma_{\mathrm{g}}^{-}, v>0\right)$ photoproducts:

$$
C=C_{\mathrm{O}}+C_{\mathrm{O}_{2}^{*}}=\frac{C_{0, \mathrm{O}}}{\sqrt{1+\left(\omega_{n} \tau_{\mathrm{O}}\right)^{2}}}+\frac{C_{0, \mathrm{O}_{2}^{*}}}{\sqrt{1+\left(\omega_{n} \tau_{\mathrm{O}_{2}^{*}}\right)^{2}}}
$$

Here, $\mathrm{O}_{2}^{*}$ represents ground-state $\mathrm{O}_{2}$ in a vibrationally excited state. Thus, we can model the variation in $C$ with bath gas composition if we can quantify the associated variations of the relaxation timescales $\tau_{\mathrm{O}}$ and $\tau_{\mathrm{O}_{2}^{*}}$. We can derive expressions for these timescales by considering the subsequent collisional processes of the nascent photoproducts.

The nascent $\mathrm{O}_{2}\left(\mathrm{X}^{3} \Sigma_{\mathrm{g}}^{-}, v>0\right)$ is formed in the ground electronic state but is vibrationally excited, with $v=0$ dominating at $\lambda=620 \mathrm{~nm}, v=4-8$ dominating at $\lambda=450-500 \mathrm{~nm}$, and even higher $v$ expected to dominate for $\lambda<450 \mathrm{~nm} .^{45,46}$ This vibrational energy is transferred to translational modes of bath gas species $\mathrm{M}=\mathrm{N}_{2}, \mathrm{O}_{2}$ through $\mathrm{V}-\mathrm{T}$ energy transfer:

$$
\mathrm{O}_{2}\left(\mathrm{X}^{3} \Sigma_{\mathrm{g}}^{-}, v\right)+\mathrm{M}\left(v^{\prime}\right) \rightarrow \mathrm{O}_{2}\left(\mathrm{X}^{3} \Sigma_{\mathrm{g}}^{-}, v-1\right)+\mathrm{M}\left(v^{\prime}\right)
$$

As we described previously, ${ }^{24}$ the timescale for this quenching is given by:

$$
\tau_{\mathrm{O}_{2}^{*}}=\left(k_{\mathrm{O}_{2}^{*}-\mathrm{N}_{2}}\left[\mathrm{~N}_{2}\right]+k_{\mathrm{O}_{2}^{*}-\mathrm{O}_{2}}\left[\mathrm{O}_{2}\right]\right)^{-1}
$$

in which $k_{\mathrm{O}_{2}^{*}-\mathrm{N}_{2}}$ and $k_{\mathrm{O}_{2}^{*}-\mathrm{O}_{2}}$ are quenching rate constants in bath gas $\mathrm{N}_{2}$ or $\mathrm{O}_{2}$, respectively, and $\left[\mathrm{N}_{2}\right]$ and $\left[\mathrm{O}_{2}\right]$ are the concentrations of the corresponding bath gases.

The nascent $\mathrm{O}\left({ }^{3} \mathrm{P}\right.$ ) rapidly combines with bath gas $\mathrm{O}_{2}$ (collision frequency of $\left.\sim 10^{12} \mathrm{~s}^{-1}\right)$ to form ground state $\mathrm{O}_{3}(\tilde{\mathrm{X}})$ via reactions (R3) and (R5):

$$
\begin{aligned}
& \mathrm{O}\left({ }^{3} \mathrm{P}\right)+\mathrm{O}_{2}\left(\mathrm{X}^{3} \Sigma_{\mathrm{g}}^{-}\right) \stackrel{k_{1}}{\longrightarrow} \mathrm{O}_{3}^{\ddagger}(\tilde{\mathrm{X}}) \\
& \mathrm{O}_{3}^{\ddagger}(\tilde{\mathrm{X}}) \stackrel{k_{2}}{\longrightarrow} \mathrm{O}\left({ }^{3} \mathrm{P}\right)+\mathrm{O}_{2}\left(\mathrm{X}^{2} \Sigma_{\mathrm{g}}^{-}\right) \\
& \mathrm{O}_{3}^{\ddagger}(\tilde{\mathrm{X}})+\mathrm{M} \stackrel{k_{3}}{\longrightarrow} \mathrm{O}_{3}(\tilde{\mathrm{X}}, v>0)
\end{aligned}
$$

in which $\mathrm{M}\left(\mathrm{N}_{2}\right.$ or $\left.\mathrm{O}_{2}\right)$ is a third body that is needed to remove energy from the unstable $\mathrm{O}_{3}^{\ddagger}(\tilde{\mathrm{X}})$ that has an internal energy above the energy dissociation threshold correlating to $\mathrm{O}\left({ }^{3} \mathrm{P}\right)+$ $\mathrm{O}_{2}\left(\mathrm{X}^{3} \Sigma_{\mathrm{g}}^{-}\right)$. Through reactions (R3) and (R5), the resulting $\mathrm{O}_{3}(\tilde{\mathrm{X}}, v>0)$ is in the ground electronic state but is vibrationally excited $(v>0)$. The collisional stabilisation of $\mathrm{O}_{3}^{\ddagger}(\tilde{\mathrm{X}})$ (R5) competes with the re-dissociation of $\mathrm{O}_{3}^{\dagger}(\tilde{\mathrm{X}})$ to $\mathrm{O}\left({ }^{3} \mathrm{P}\right)+\mathrm{O}_{2}\left(\mathrm{X}^{3} \Sigma_{\mathrm{g}}^{-}\right)$ (R4), with this latter process expected to be fast compared to the stabilisation pathway. The contribution to the PAS signal from $\mathrm{O}_{3}(\tilde{\mathrm{X}}, v>0)$ will depend on (i) the rate at which stabilised $\mathrm{O}_{3}(\tilde{\mathrm{X}}, v>0)$ is formed, and (ii) the rate of $\mathrm{V}-\mathrm{T}$ energy transfer from vibrationally excited $\mathrm{O}_{3}(\tilde{\mathrm{X}}, v>0)$ to translational motion of $\mathrm{N}_{2}$ and $\mathrm{O}_{2}$. Our previous work discussed that, for measurements at equilibrium pressures $>950 \mathrm{hPa}$, the latter process is sufficiently fast that we need only consider the rate at which vibrationally-stabilised $\mathrm{O}_{3}(\tilde{\mathrm{X}}, v>0)$ is formed. ${ }^{24}$ Applying the steady state approximation to reactions (R3)-(R5), the time dependence in the loss of $\mathrm{O}\left({ }^{3} \mathrm{P}\right)$, and the formation of $\mathrm{O}_{3}(\tilde{\mathrm{X}}, v>0)$ follows an exponential form with a time constant:

$$
\tau_{\mathrm{O}}=\left(k_{\mathrm{O}-\mathrm{O}_{2}-\mathrm{N}_{2}}\left[\mathrm{O}_{2}\right]\left[\mathrm{N}_{2}\right]+k_{\mathrm{O}-\mathrm{O}_{2}-\mathrm{O}_{2}}\left[\mathrm{O}_{2}\right]^{2}\right)^{-1}
$$

in which $k_{\mathrm{O}-\mathrm{O}_{2}-\mathrm{N}_{2}}$ and $k_{\mathrm{O}-\mathrm{O}_{2}-\mathrm{O}_{2}}$ are rate coefficients related to the formation rate for $\mathrm{O}_{3}^{\ddagger}(\tilde{\mathrm{X}})\left(k_{1}\right)$, the $\mathrm{O}_{3}^{\ddagger}(\tilde{\mathrm{X}})$ re-dissociation rate $\left(k_{2}\right)$, and the quenching rate of $\mathrm{O}_{3}^{\ddagger}(\tilde{\mathrm{X}})$ by $\mathrm{M}$ to form $\mathrm{O}_{3}(\mathrm{X}, v>0)\left(k_{3}\right)$, for $\mathrm{N}_{2}$ or $\mathrm{O}_{2}$ bath gas species respectively.

Using this photochemical model (eqn (6)-(8)), we reproduced our measured variations in $C$ with bath gas composition and wavelength. ${ }^{24}$ We recommended that PAS calibrations are performed in a bath gas of air $\left(x_{\mathrm{O}_{2}} \sim 0.2-0.3\right)$. The reduced sensitivity in the limit of pure $\mathrm{N}_{2}$ corresponded to slow recombination of $\mathrm{O}\left({ }^{3} \mathrm{P}\right)$ with bath gas $\mathrm{O}_{2}$, with eqn (8) demonstrating the energy quenching timescale $\tau_{\mathrm{O}}$ tends to infinity as $\left[\mathrm{O}_{2}\right] \rightarrow 0$. Meanwhile, the reduced sensitivity as $\left[\mathrm{O}_{2}\right] \rightarrow 1$ was attributed to a lower $\mathrm{V}-\mathrm{T}$ quenching rate for $\mathrm{O}_{2}(\mathrm{X}, v>0)$ by bath gas $\mathrm{O}_{2}$ compared to that by $\mathrm{N}_{2}$.

\subsection{Remaining knowledge gaps}

The above discussion has shown that quantifying energy transfer timescales for the nascent $\mathrm{O}\left({ }^{3} \mathrm{P}\right)$ and $\mathrm{O}_{2}\left(\mathrm{X}^{3} \Sigma_{\mathrm{g}}^{-}, v>0\right)$ photoproducts is crucial to assess the fidelity of visiblewavelength photoacoustic methods for detection of $\mathrm{O}_{3}$. We acknowledge two deficiencies in our earlier assessments of these energy transfer timescales. First, our previous work demonstrated that fitting the above photochemical model to measured variations in $C$ with bath gas composition constrained poorly the relative rates of quenching of the $\mathrm{O}_{3}^{+}$ complex by either $\mathrm{N}_{2}$ or $\mathrm{O}_{2}$ bath gas. Second, we need to quantify the dependencies of the energy transfer timescales $\tau_{\mathrm{O}_{2}^{*}}$ and $\tau_{\mathrm{O}}$ on pressure. During photoacoustic measurements, such as from an atmospheric research aircraft (our application area), the equilibrium sample pressure can vary over the range 300-1000 hPa and we need to quantify the dependence of $C$ on equilibrium (static) pressure. Microphones have a pressure dependent response function, ${ }^{7,47}$ since the motion of the microphone membrane is progressively damped as static pressure increases. Our experience shows that this response function can change over time and vary between microphones. This response function needs to be corrected for during postprocessing of PAS measurements, yet this correction is often neglected. ${ }^{38,40}$ In recent years, we have sought to correct for this 
pressure-dependent microphone response using our ozonebased calibration approach to measure $C$ at different static pressure levels within the range $300-1000 \mathrm{hPa} .{ }^{15}$ This approach assumes that there is no pressure-dependent quenching of the ozone photoproducts and that pressure dependence arises only from changes in the microphone response function. While this approach might seem logical without prior appreciation of the ozone photoproduct quenching timescales, we show in this work that there are fundamental issues associated with this calibration approach and describe a new measurement strategy for determining pressure-dependent microphone response functions.

This work addresses the two aforementioned knowledge gaps by performing measurements of the variation in $C$ with bath gas composition, wavelength and static pressure. Moreover, for the first time, we report the associated variations in the PAS phase shift $\theta$ (eqn (3)). This phase shift provides the much-needed additional constraint on the associated rate constants. The following section describes in detail the photoacoustic transduction processes that depend on static pressure, and describes a measurement approach for characterising pressure dependence in the microphone response function that enables us to study the relaxation timescales of Chappuis band photoproducts at reduced pressures. Section 3 describes the experimental methods used in this work, for both the characterisation of pressure dependent microphone response functions and measurements of $C$ and $\theta$ for $\mathrm{O}_{3}$-laden samples. Then, Section 4 reports our measurement results, including a comparison of measured variations in $C$ and $\theta$ with predictions from our ozone photochemical model.

\section{Pressure-dependent processes in photoacoustic measurements}

We now consider how the static pressure $P_{0}$ affects various stages of the photoacoustic transduction process and approaches to correcting for static pressure effects. Fig. S1-1 in the ESI $\dagger$ summarises this transduction and highlights the pressure dependent processes. We will initially assume that the analyte behaves ideally, i.e. energy transfer from the analyte to bath gas translational modes is always fast and thus $\omega_{n} \tau \ll 1$. This assumption is valid for typical non-volatile aerosols of submicrometre diameter at ambient pressure. ${ }^{26,27}$ Using the model of Chan, ${ }^{48}$ the characteristic cooling time for black carbon particles with an effective spherical diameter of $1 \mu \mathrm{m}$ (i.e. the largest particle size measured with our instruments) increases from $4.8 \mu \mathrm{s}$ for $P_{0}=1000 \mathrm{hPa}$ to $5.1 \mu \mathrm{s}$ for $P_{0}=300 \mathrm{hPa}$. These timescales are much shorter than the 620-660 $\mu$ s modulation timescales for our PAS instruments. Furthermore, the aerosol cooling timescales reduce significantly for smaller aerosol diameters $D$; a $300 \mathrm{~nm}$ diameter (a typical median diameter for our measurements) black carbon particle has a cooling timescale of $\sim 0.4-0.5 \mu \mathrm{s}$.

Assuming efficient energy transfer to bath gas translational modes, the absorbing sample is homogeneously distributed within the PAS cell, and single eigenmode excitation of the PAS cell at the corresponding resonance frequency, the photoacoustic pressure at the location of the microphone is given by eqn (2). In this expression, both $Q_{n}$ and $\omega_{n}$ depend on the density of the bath gas and, therefore, on $P_{0} \cdot{ }^{7,38}$ There is also a small dependence of the adiabatic coefficient on $P_{0}$, but the factor $\sigma-1$ varies by less than $0.5 \%$ over the pressure range of interest in this work (300-1000 hPa). Meanwhile, the terms $I_{0}$, $g(\vec{r}), V$, and $p_{n}(\vec{r})$ are independent of $P_{0}$. By definition, the sample absorption coefficient will decrease with the absorber concentration that inherently scales with pressure, although measurements of the PAS sensitivity $C$ are normalised for the independently measured sample absorption (eqn (4)). Similarly, the impacts of pressure broadening on the spectral linewidths of molecular absorption bands are corrected for in measurements of $C$. Thus, ignoring variations in $\alpha_{\mathrm{abs}}$, the normalised photoacoustic signal $\left|p\left(\vec{r}_{\mathrm{M}}\right)\right| /\left(Q_{n} / \omega_{n}\right)$ is independent of $P_{0}$. We validated the above transduction model using a finite element method (FEM) model. We have described our FEM model in previous publications ${ }^{30,31}$ that predicts $\left|p\left(\vec{r}_{\mathrm{M}}\right)\right|$ for our PAS cells. Fig. S1-2(a) in the ESI $\dagger$ shows the FEM-predicted $\left|p\left(\vec{r}_{\mathrm{M}}, \omega\right)\right|$ with variation in the PAS modulation frequency for four different $P_{0}$ values over the range 400-1000 hPa. The photoacoustic pressure maximum at resonance $\left|p\left(\vec{r}_{\mathrm{M}}, \omega_{n}\right)\right|$ decreases with a reduction in $P_{0}$, while Fig. S1-2(c) (ESI $\dagger$ ) shows that the normalised signal $\left|p\left(\vec{r}_{\mathrm{M}}, \omega_{n}\right)\right| /\left(Q_{n} / \omega_{n}\right)$ is indeed independent of $P_{0}$.

The amplified photoacoustic wave drives motion of a microphone diaphragm. We used miniature electret microphones in which the diaphragm is composed of a dielectric material with a permanent electric dipole moment. Motion of this diaphragm with respect to a stationary back plate drives a change in capacitance. A small perforation in the diaphragm allows the static pressure to equalise across this membrane. The output microphone voltage $V_{\text {mic }}$ is then proportional to the photoacoustic pressure $p\left(\vec{r}_{\mathrm{M}}\right)$ at the microphone surface that drives displacement of the microphone diaphragm:

$$
V_{\text {mic }}(t)=k_{\mathrm{M}} p\left(\vec{r}_{\mathrm{M}}\right)
$$

$k_{\mathrm{M}}$ is a microphone response function and depends on - among other factors concerned with the pre-amplification of the electrical signal - the permanent voltage and equilibrium distance between the dielectric membrane and back plate, the diaphragm properties (surface area, mass, tension), and the damping ratio which depends on the density of the bath gas. Mechanical models often treat microphone diaphragm motion as a driven harmonic oscillator. ${ }^{49,50}$ The damping ratio $\zeta$ governs the damping regime of this motion, with $\zeta=1$ corresponding to critical damping and smaller or larger $\zeta$ values indicating under or over-damping, respectively. The damping ratio is inversely proportional to the diaphragm mass and for miniaturised microphones, with inherently low diaphragm masses, their diaphragm motion has been shown to exhibit over-damped to critically-damped response characteristics. ${ }^{51}$ In this regime, the magnitude of the drag coefficient is key in determining the overall microphone 
response function at different static pressures. Characterisations of pressure-dependent response functions are limited for miniature electret microphones. For a one-inch diameter condenser microphone, Thomas et al. measured a reduction in response function at elevated pressures attributed to increased viscous damping of the microphone diaphragm motion at higher pressures. ${ }^{47}$

Our challenge is to correct the recorded microphone voltage for the pressure dependent processes above and arrive at a quantity that is directly proportional to $\alpha_{\text {abs }}$. It is straightforward to correct $V_{\text {mic }}$ for variations in the PAS cell amplification by dividing $V_{\text {mic }}$ by $Q_{n} / \omega_{n}$; Section 3 describes our measurements of $Q_{n}$ and $\omega_{n}$ whenever the bath gas density changes (through variations in $P_{0}$ or composition) by exciting PAS cell eigenmodes using a speaker transducer. To correct for the $P_{0}$-dependence of the microphone response function $k_{\mathrm{M}}$ we used a measurement approach similar to Arnott et al. to characterise the relative variation in $k_{\mathrm{M}}$ with $P_{0}{ }^{7}$ Arnott and co-workers measured the microphone response to the pressure field induced by a piezoelectric transducer. The transduction pathway for generating a microphone response from excitation of the PAS cell using a speaker transducer is different to that for photoacoustic transduction, because the speaker transducer directly drives periodic displacement of the bath gas. The acoustic pressure amplitude $\left|p_{\text {spk }}\right|$ immediately in front of the speaker diaphragm is related to the amplitude of the diaphragm displacement amplitude $x_{\mathrm{spk}}$ by: $^{52}$

$$
\left|p_{\mathrm{spk}}\right|=\rho v \omega x_{\mathrm{spk}}
$$

in which $\rho$ is the bath gas density (that depends on $P_{0}$ and bath gas composition), and $v$ is the speed of sound (which varies with bath gas composition only). The PAS cell amplifies the speaker-generated pressure wave by a factor proportional to $Q_{n} / \omega_{n}$. Therefore, the pressure at the microphone surface when exciting the cell on resonance is described by:

$$
\left|p\left(\vec{r}_{\mathrm{M}}\right)\right|=B \rho v Q_{n} x_{\mathrm{spk}}
$$

in which $B$ is a pressure-independent constant of proportionality related to the PAS cell eigenmode pressure distribution and the location of the microphone. Combining eqn (9) and (11), we see that the microphone response function is described by:

$$
k_{\mathrm{M}}=\frac{V_{\mathrm{mic}}}{B \rho v Q_{n} x_{\mathrm{spk}}}
$$

After accounting for the impact of $P_{0}$ on $\rho$ and $Q$, Arnott et al. reported that the response function for their one-inch condenser microphone was invariant over the $P_{0}$ range 500-1000 $\mathrm{hPa}^{7}$ Using the same measurement approach, we will show that significant variations in $k_{\mathrm{M}}$ with $P_{0}$ are found for our miniature electret microphones that indicate increased damping of our microphone diaphragms at higher pressures. In measuring the relative variation in $k_{\mathrm{M}}$ with $P_{0}$, we assume that $x_{\mathrm{spk}}$ is independent of $P_{0}$. We acknowledge that the speaker displacement could vary with $P_{0}$ depending on the damping regime of the speaker membrane. However, we will justify our approach by demonstrating that the determined variations in $k_{\mathrm{M}}$ suitably correct absorption data for monodisperse laboratory-generated aerosol standards to provide the expected pressure-invariance in the measured aerosol coalbedo.

\section{Experimental methods}

Section 3.1 describes pertinent aspects to our cavity ring-down spectroscopy (CRDS) and PAS measurements. Section 3.2 describes our methods for measuring aerosol absorption with variation in $P_{0}$ for aerosol standards that provide data sets for characterising pressure-dependent microphone responses. Then, Section 3.3 outlines our methods for measuring $C$ and $\theta$ using $\mathrm{O}_{3}$-laden gas samples at controlled pressures, bath gas compositions and for varying photolysis wavelengths.

\subsection{Photoacoustic and cavity ring-down instruments}

Our previous publication described the construction and flow configuration of our CRDS and PAS instruments, and the associated calibration of our PAS instruments using $\mathrm{O}_{3}$-laden gas samples. ${ }^{24}$ We only summarise our instruments here and highlight differences to our previous work. One difference is that the PAS instruments used in this work used an optimised two-resonator photoacoustic cell that we described recently and that maximises the sensitivity of photoacoustic detection. ${ }^{31}$ Fig. 1(a) summarises the arrangement and flow configuration for the spectrometers. Our PAS instruments operated at wavelengths $\lambda=405,514,658 \mathrm{~nm}$, while our CRDS instruments used $\lambda=405$, $658 \mathrm{~nm}$ only. Sample flow was drawn through three parallel flow lines, with each line corresponding to a different spectroscopy wavelength. When both a CRDS and PAS spectrometer were available at the same wavelength, the CRDS and PAS instrument were arranged in a serial flow configuration such that the sample was drawn first through the CRDS instrument. Mass flow controllers (MFCs) regulated the volumetric sample flow rate drawn through each flow line to a set-point of $1 \mathrm{~L} \mathrm{~min}{ }^{-1}$. The sample was drawn through a common inlet, before passing through a Nafion dryer and an activated charcoal scrubber to remove trace $\mathrm{NO}_{x}$ and $\mathrm{O}_{3}$ gases that absorb light at our spectroscopy wavelengths. Automated valves controlled whether the sample was routed through a HEPA filter prior to passing through the spectrometers. This filtering stage removed aerosol to enable background measurements required for characterising the empty-cavity ring-down time (for the CRDS) and background microphone response (for the PAS). For measurements of $C$ and $\theta$ using $\mathrm{O}_{3}$-laden gas samples, an ozonized oxygen flow (i.e. an $\mathrm{O}_{3}-\mathrm{O}_{2}$ mixture) was diluted into the conditioned sample flow immediately prior to our spectrometers.

A speaker transducer excited PAS cell eigenmodes to enable quantification of $Q_{n}$ and $f_{n}$, in addition to changes in $k_{\mathrm{M}}$. A speaker was located close to the centre of the lower resonator of our two-resonator cell. This speaker was driven by a voltage waveform that, in the frequency domain, had a top hat distribution over the frequency range $1300-1700 \mathrm{~Hz}$. This acoustic 
(a) Spectroscopy measurements

$\bigotimes$ Automated valve

* sample inlet

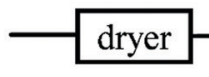

scrubbe

P) Pressure sensor

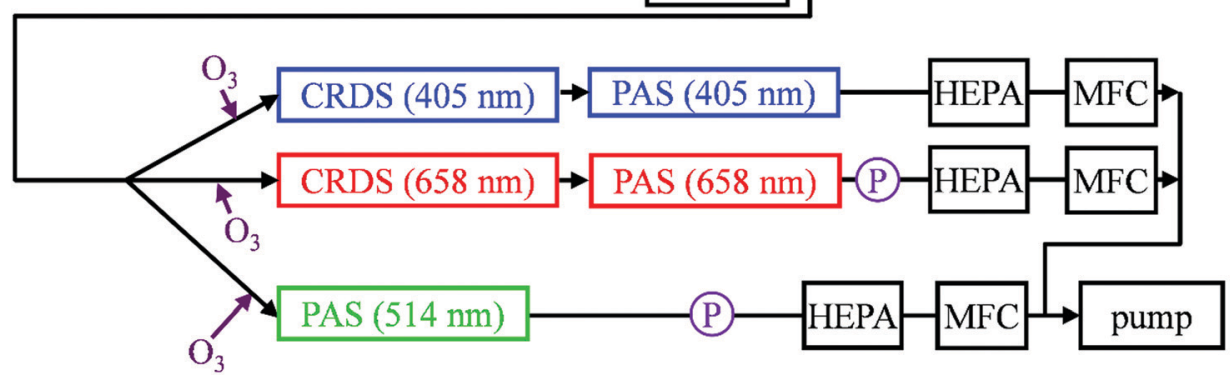

(b) Pressure control for aerosol sample

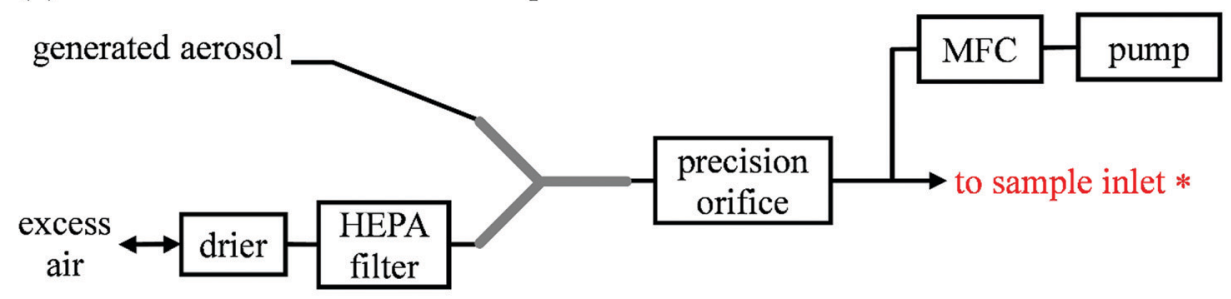

(c) Pressure control for bath gas during $\mathrm{O}_{3}$ calibrations

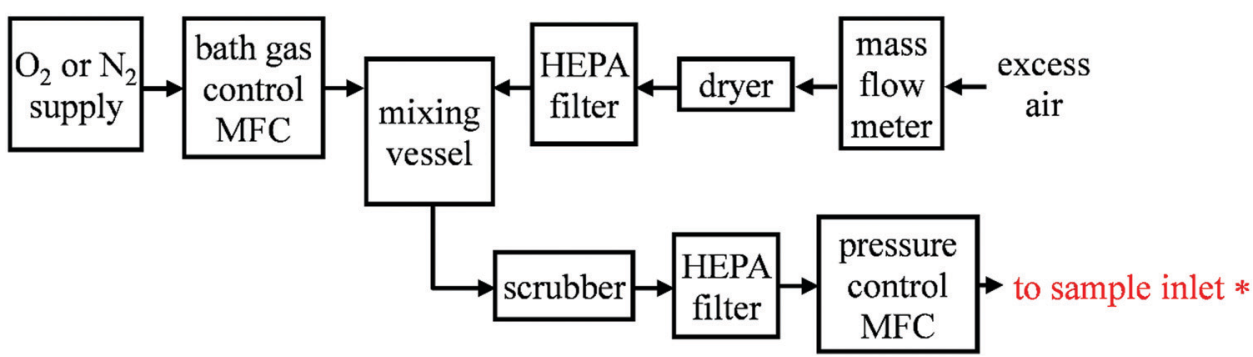

Fig. 1 Schematic diagrams showing: (a) the flow configuration for drawing sample through our spectroscopic instruments; (b) the control of $P_{0}$ for samples containing mobility-selected aerosol using a precision orifice; (c) the control of $P_{0}$ for the bath gas using a pressure controller during calibrations of our PAS instruments with an $\mathrm{O}_{3}$ laden gas sample. MFC denotes a mass flow controller, HEPA filter denotes a high efficiency particle absorbing filter, pressure sensors correspond to Honeywell PPT-series pressure transducers.

spectral range extended across the eigenmode spectral width. Speaker-excitation of the PAS cell was performed immediately prior to any measurements of light-absorbing aerosol or $\mathrm{O}_{3}$-laden gas, and after any changes in the bath gas density (through changes in either $P_{0}$ or composition). During the speaker excitation period (of $30 \mathrm{~s}$ duration), the microphone waveform was recorded in $1 \mathrm{~s}$ intervals, with each waveform processed through a fast Fourier transform that gave the power spectrum for the eigenmode excitation. A Lorentzian function was fitted to this power spectrum to retrieve $Q_{n}, f_{n}$ and the peak signal amplitude (denoted $S_{\text {spk }}$ ). A software-controlled feedback used the determined $f_{n}$ to set the laser power modulation frequencies for each PAS instrument.

Several sensors were integrated into the flow system to measure $P_{0}$. The MFCs regulating the volumetric flow rates for each flow line provided pressure readings. However, these MFCs were located downstream of a HEPA filter (see Fig. 1(a)), and a small pressure drop across this filter reduced the pressure at the MFCs. Instead, separate Precision Pressure Transducers (PPTs, Honeywell PPT-series) were located immediately after the 514 and 658 nm PAS instruments. Fig. S2-1 in the ESI $\dagger$ compares the pressures recorded by our five pressure sensors and demonstrates the reduced pressures recorded by the three MFC sensors compared to those by the two PPT sensors. In principle, the PPT sensors should record the same pressure value and represent the sample pressure in all spectrometers; there were no elements of the flow system that would lead to differences in $P_{0}$ between spectrometers. The pressure recorded by the PPT sensor located in the $658 \mathrm{~nm}$ sample flow line was consistently higher by $3.3 \pm 0.5 \mathrm{hPa}$ compared to that 
in the $514 \mathrm{~nm}$ line. We took the mean $P_{0}$ provided by these two pressure sensors as representing the pressure in all five spectrometers.

\subsection{Measurements of aerosol absorption at reduced pressure for aerosol standards}

Aerosol coalbedo (the ratio of the absorption to extinction coefficient) is independent of pressure. Moreover, Section 2 showed that sub-micron non-volatile aerosols can be considered to liberate their heat to the surrounding bath gas instantaneously on our PAS modulation timescales and over the $P_{0}$ range 300-1000 $\mathrm{hPa}$. Thus, measurements of the coalbedo from PAS-measured aerosol absorption coefficients in combination with CRDS-measured extinction coefficients should be invariant with $P_{0}$ if the microphone response function is independent of static pressure. Providing that the size distribution for the sampled aerosol is not affected by changes in $P_{0}$, any discrepancy between the measured coalbedo at ambient pressure and that at reduced $P_{0}$ is attributed to changes in $k_{\mathrm{M}}$.

In these measurements, our spectrometers sampled laboratory generated light absorbing aerosol standards at different $P_{0}$. Our aerosol generation and conditioning methods follow closely the methods we reported in ref. 22 , to which we refer the reader for specifications of equipment used. We used two aerosol standards; monodisperse dyed PolyStyrene Latex (PSL) spheres or nigrosin dye aerosols.

In the PSL measurements, an aqueous stock solution containing $250 \mathrm{~nm}$ diameter PSL spheres (Thermo Scientific, Custom Black polystyrene microspheres in water, CAT No. SAM423) was passed to the input of a constant output atomiser. A constant flow of high purity $(99.999 \%)$ zero air from a gas cylinder with an outlet pressure of $2400 \mathrm{hPa}$ generated aqueous aerosol that passed through two indicating silica gel diffusion driers that dried the aerosol sample to a relative humidity of $<5 \%$, as determined by a humidity probe placed immediately after the second drier. Fig. 1(b) shows how we regulated the pressure of the aerosol sample. The dried sample passed to an input of a ' $\mathrm{Y}$ ' flow splitter (Brechtel Manufacturing Inc.), with our CRDS-PAS instruments drawing aerosol sample (via a precision orifice) from one exit port of this splitter, while the other port was connected to a filter and drier (containing indicating silica gel desiccant) to dump excess sample. The aerosol-laden flow to the CRDS-PAS passed through a constricted precision orifice (O'Keefe Controls Co.); we used a range of orifice diameters to control the output sample pressure, with orifice diameters in the range 0.51-0.69 $\mathrm{mm}$. This orifice created a pressure drop, with the static pressure at the orifice output determined by the orifice diameter and the volumetric flow rate through the orifice. The output of this orifice was connected, via a ' $\mathrm{T}$ ' flow splitter (also referred to commonly as a 'union Tee'), to the common sample inlet of our CRDS-PAS instruments. A further MFC (referred to as the 'dump MFC') was connected to the second output from the ' $\mathrm{T}$ ' splitter and was used to draw sample immediately after the constricted orifice to an exhaust pump. By controlling the orifice diameter and the dump MFC flow rate, we could vary $P_{0}$ of the aerosol-laden sample over the range $300-1000 \mathrm{hPa}$. The aerosol extinction and absorption coefficients were recorded at $1 \mathrm{~Hz}$ sampling frequencies for 5 minutes at each $P_{0}$. At each pressure level and prior to sampling aerosol-laden air samples, we recorded the CRDS empty cavity ring-down times and the background microphone responses enabled through the automated routing of the sample through a HEPA filter prior to spectroscopic measurements. The particulate filter used during these measurements (Headline Filters, DIF-LK40) had a large filter area that created a minimal sample pressure drop $(\sim 2.5 \mathrm{hPa})$. Furthermore, we used speaker excitation of the PAS cell to characterise $S_{\mathrm{spk}}, Q_{n}$ and $f_{n}$ at each $P_{0}$. Again, we refer the reader to our recent publication for details of the post-processing of the raw spectroscopy data, calibration of the PAS instruments using $\mathrm{O}_{3}$-laden air, and assessments of the accuracy of our extinction and absorption measurements. ${ }^{22}$

The measurements using nigrosin aerosol were similar to those using dyed PSL spheres. The aqueous stock solution supplying the atomiser contained water soluble nigrosin dye (Sigma Aldrich, CAS number 8005-03-6, lot number MKBR1705V) with a solute concentration of $2 \mathrm{~g} \mathrm{~L}^{-1}$. To prevent complete attenuation of the intra-cavity ring-down laser beam, we controlled the aerosol number concentration by passing the aerosol-laden flow through a differential mobility analyser (DMA) prior to the ' $\mathrm{Y}$ ' flow splitter in Fig. 1(b). The DMA was set to pass aerosol particles with a mobility-selected diameter of $200 \mathrm{~nm}$. The DMA sheath and aerosol sample flows were 1.0 and $0.61 \mathrm{~L} \mathrm{~min}^{-1}$ respectively, with the sheath flow set deliberately to a low value such that the DMA transfer function was wide enough to pass a high particle number concentration for maximising the signal-to-noise ratio in extinction and absorption measurements, but remained sufficiently narrow to prevent complete attenuation of our intra-cavity ring-down laser beam. A final distinguishing factor between the PSL and nigrosin measurements was that, in the case of nigrosin, the dump MFC was not required and the pressure drop was controlled by varying the diameter of the precision orifice only over the range $0.46-0.89 \mathrm{~mm}$.

\subsection{Sensitivity and phase shift measurements for $\mathrm{O}_{3}$-laden gas samples at reduced pressures}

We measured $C$ and $\theta$ for $\mathrm{O}_{3}$ added to bath gases composed of various $\mathrm{O}_{2}-\mathrm{N}_{2}$ mixtures at different static pressures. We describe only the differences to the methods we reported in ref. 24. Specifically, we discuss the control of $P_{0}$ during measurements (which was not varied in previous work), and measurements of $\theta$ (which was not investigated previously).

Fig. 1(c) shows how we controlled the bath gas composition and pressure during measurements of $\mathrm{O}_{3}$-laden samples, while Fig. 1(a) indicates the locations at which an ozonized- $\mathrm{O}_{2}$ gas flow was diluted into the pressure-controlled bath gas. We regulated the mass flow of $\mathrm{O}_{2}$ or $\mathrm{N}_{2}$ from a high purity (99.999\%) gas cylinder into a $1.1 \mathrm{~L}$ aluminium mixing vessel, in which the high purity gas was mixed with ambient air $(\sim 21 \%$ $\mathrm{O}_{2}, 78 \% \mathrm{~N}_{2}, 1 \%$ argon and trace amounts of other gases) drawn 
through an in-line dryer (containing indicating silica gel desiccant) and a HEPA filter. A mass flow meter monitored the mass flow rate of ambient air into the mixing volume. By changing the mass flow rate of the high purity gas into the mixing volume, we varied the $\mathrm{O}_{2}$ mass fraction of the bath gas over the range 0.0-1.0. The mixed gas was then drawn through a Purafil-containing gas scrubber to remove trace $\mathrm{NO}_{x} / \mathrm{O}_{3}$ species, before passing through a further HEPA filter. The bath gas passed to the common sample inlet (indicated in Fig. 1(a)) via a pressure controller (Alicat PC Series) that regulated the bath gas pressure. We measured $C$ and $\theta$ for five values of $\mathrm{O}_{2}$ mass fraction $\left(x_{\mathrm{O}_{2}}=0.02,0.05,0.25,0.63,1.00\right)$ and five values of static pressure $\left(P_{0}=1007.5,901.9,702.5,503.1,304.1 \mathrm{hPa}\right)$. The specified values for $x_{\mathrm{O}_{2}}$ account for small contributions to the bath gas compositions from the CRDS mirror purge flows and the $\mathrm{O}_{2}$ contribution from the ozonized oxygen calibration gas. The $P_{0}$ values chosen were informed by our experience from our research aircraft measurements, during which the ambient pressure approaches $300 \mathrm{hPa}$ at altitudes close to $10 \mathrm{~km}$.

The measurements of $C$ are corrected for the impacts of both $P_{0}$ and bath gas composition on the amplification characteristics of the PAS cell $\left(Q_{n}\right.$ and $\left.f_{n}\right)$, absorber concentration, and small variations in the intra-cavity laser power, in addition to changes in microphone response $k_{\mathrm{M}}$ using the new approach described in Section 2 and validated in Section 4.1. Thus, any variations in the measured $C$ presented here with either $P_{0}$ or bath gas are attributed to changes in the rates for energy transfer from the nascent photofragments following $\mathrm{O}_{3}$ photolysis to bath gas translational modes. The phase shift provides a more direct measurement of relaxation timescales in photoacoustic processes compared to $C$. We define $\theta$ as the difference in phases recorded for the microphone waveform and that for the sinusoidal waveform used to drive the laser power modulation. Section S3 of the ESI $\dagger$ describes how these individual phase shifts are acquired and how $\theta$ is processed. Specifically, no correction for microphone response, PAS cell amplification characteristics or small drifts in laser power are needed, although a background instrument phase shift subtraction is required in addition to a correction for small $(\leq 0.5 \mathrm{~Hz})$ mismatches between the laser modulation frequency and that of the PAS cell eigenmode, both of which are straightforward corrections (see Section S3 of the ESI $\dagger$ ).

\section{Results and discussion}

4.1 Validation of approach to measuring relative variations in $\boldsymbol{k}_{\mathrm{M}}$ with changes in $\boldsymbol{P}_{\mathbf{0}}$

Measurements of coalbedo for benchmark light absorbing aerosols at different $P_{0}$ enabled us to validate our approach to characterising pressure dependent microphone responses as described in Section 2. To provide a metric for the fractional change in measured aerosol coalbedo with $P_{0}$, we define the relative coalbedo as the ratio of the coalbedo to that measured at the highest pressure $\left(P_{0} \sim 1000 \mathrm{hPa}\right)$. Initially, the absorption measurements are corrected only for the $P_{0}$ dependences of $Q_{n}, f_{n}$ and small variations in the laser power. Fig. 2(a) shows the variation in relative coalbedo with $P_{0}$ for both dyed PSL and nigrosin aerosols. The extinction measured by the $658 \mathrm{~nm}$ CRDS instrument was used in calculating the relative coalbedo for all channels as these $658 \mathrm{~nm}$ extinction measurements were superior in precision compared to those recorded by the $405 \mathrm{~nm}$ CRDS instrument. This approach is sound and the exact value for coalbedo is unimportant; simply, we are using the extinction measurements to normalise the PAS-measured absorption values for the reduction in aerosol number concentration that occurs with a reduction in pressure. We recognise that we could have used a condensation particle counter to measure these changes in number concentration, thus enabling measurements of aerosol absorption cross sections that are pressure-independent quantities. However, the particle counters available in our laboratory operate only at pressures in the range 750-1050 hPa.

For both nigrosin and PSL aerosol measurements, the measured coalbedo increases by $30-45 \%$ as $P_{0}$ is reduced from 1000 to $300 \mathrm{hPa}$. As these coalbedo measurements are already corrected for pressure dependence in $Q_{n}$ and $f_{n}$, in addition to small variations in laser power, the remaining pressure dependence is attributed to changes in $k_{\mathrm{M}}$. Using the approach described in Section 2, speaker excitation measurements of $Q_{n}$ and $S_{\text {spk }}$ are used to characterise the pressure dependent microphone response function. From eqn (12), we can define a correction factor $k_{\mathrm{CF}}$ as the ratio $k_{\mathrm{M}}$ to that at $P_{0}=1000 \mathrm{hPa}$ :

$$
k_{\mathrm{CF}}=\frac{k_{\mathrm{M}}\left(P_{0}\right)}{k_{\mathrm{M}}\left(P_{0}=1000 \mathrm{hPa}\right)}=\frac{10^{3} S_{\mathrm{spk}} Q_{n}^{\ominus}}{P_{0} S_{\mathrm{spk}}^{\ominus} Q_{n}}
$$

in which we have used $S_{\mathrm{spk}}$ instead of the microphone voltage amplitude $V_{\text {mic }}$, while $Q_{n}^{\ominus}$ and $S_{\mathrm{spk}}^{\ominus}$ represent the $Q_{n}$ and $S_{\mathrm{spk}}$ at standard pressure $\left(P_{0}=1000 \mathrm{hPa}\right)$. In writing the right-hand side of this equation, we assume that $x_{\mathrm{spk}}$ is independent of pressure, recognise that $B$ and the speed of sound are invariant with pressure, recall that the density of an ideal gas scales linearly with $P_{0}$, and define $P_{0}$ in units of hPa. Fig. 2(b) shows the measured variations in $k_{\mathrm{CF}}$ for the $405 \mathrm{~nm}, 514 \mathrm{~nm}$, and $658 \mathrm{~nm}$ PAS instruments, which exhibit similar trends with $P_{0}$ as observed for the relative coalbedo. Thus, we used $k_{\mathrm{CF}}$ to correct the PAS-measured absorption coefficients by dividing the absorption values by the mean $k_{\mathrm{CF}}$ at each pressure level. Fig. 2(c) shows the corrected relative coalbedo measurements for both dyed PSL and nigrosin aerosol, demonstrating that the measured coalbedo is invariant with $P_{0}$ when the pressuredependent microphone response is accounted for. The mean value for relative coalbedo is $0.951 \pm 0.035$, indicating systematic biases of up to $5 \%$ arise from measurements at reduced pressure, although Fig. 2(c) shows that such systematic errors are similar in magnitude to the precision with which coalbedo is measured. This result vindicates our PAS absorption correction scheme that uses speaker excitation of the PAS cell to characterise relative changes in microphone sensitivity with $P_{0}$. This method of correcting for $k_{\mathrm{M}}$ has the advantage that regular and fast characterisations of the photoacoustic 


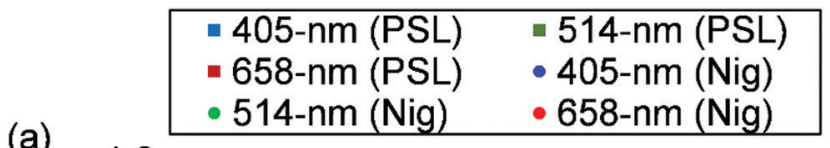

(a)

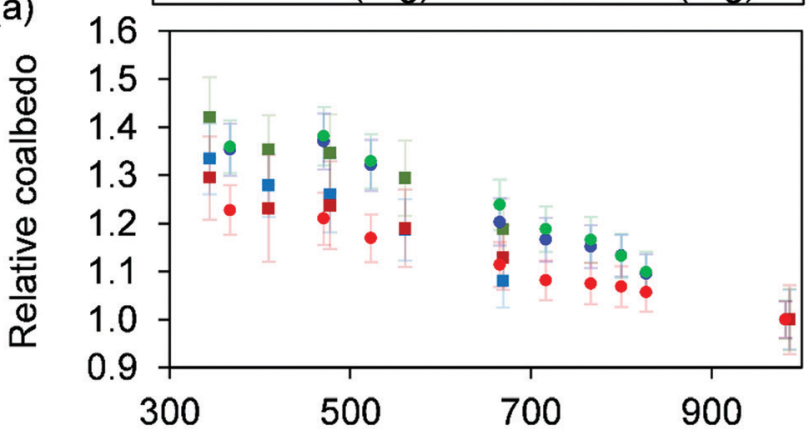

(b)
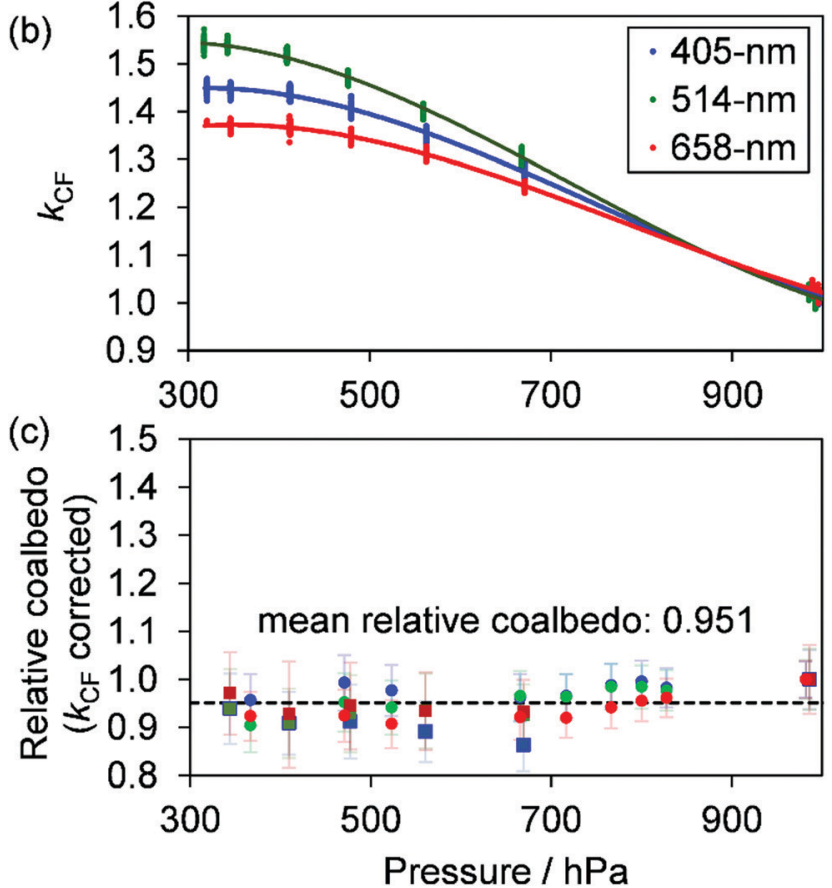

Fig. 2 (a) Measured coalbedo values relative to that measured at $P_{0} \sim$ $1000 \mathrm{hPa}$ for either $250 \mathrm{~nm}$ dyed PSL spheres or $200 \mathrm{~nm}$ mobility-selected nigrosin aerosol. Data are shown for measurements using the 405, 514 and $658 \mathrm{~nm}$ wavelength PAS instruments. (b) The relative variation in $k_{\mathrm{CF}}$ with $P_{0}$ for speaker-excitation of each PAS cell (lines are to guide the eye only). (c) The variation in relative coalbedo with $P_{0}$ for the same PSL and nigrosin aerosol samples as in (a), but with the PAS-measured absorption corrected for $k_{C F}$. The black dashed line indicates the mean value for the corrected relative coalbedo.

response (via speaker-excitation measurements of $Q_{n}$ and $S_{\mathrm{spk}}$ ) can be made without the need for controlled generation of a test aerosol sample.

\subsection{The impact of $P_{0}$ and bath gas composition on the energy transfer timescales from Chappuis band photoproducts}

Here, we present measurements of $C$ and $\theta$ from PAS measurements on $\mathrm{O}_{3}$-laden gas samples and demonstrate that characterising relative variations in $k_{\mathrm{M}}$ is critical to quantifying accurately the impacts of slow $\mathrm{O}_{3}$ photoproduct energy transfer rates on the photoacoustic signal. Notably, we show that $k_{\mathrm{CF}}$ depends on the ratio of $\mathrm{N}_{2}$ and $\mathrm{O}_{2}$ in the bath gas, even at fixed pressure, and accounting for this dependence revises our previous recommendations for accurate calibrations of PAS instruments at visible wavelengths using $\mathrm{O}_{3}$-laden gas samples. ${ }^{24}$

Our previous publication demonstrated that the quenching timescales for energy transfer from Chappuis band $\mathrm{O}_{3}$ photoproducts to the bath gas can be similar to our photoacoustic laser modulation period for some bath gas compositions, causing a negative bias in measurements of $C .{ }^{24}$ We measured this negative bias for bath gas mixtures of $\mathrm{N}_{2}$ and $\mathrm{O}_{2}$ for pressures $P_{0} \sim 1000 \mathrm{hPa}$ only. However, our photochemical model predicts this negative bias to worsen at reduced pressures. Section 1.3 summarised the photochemistry of $\mathrm{O}_{3}$ and the relaxation of the nascent photoproducts following Chappuis band photoexcitation. The timescales of energy transfer from the $\mathrm{O}_{2}\left(\mathrm{X}^{3} \Sigma_{\mathrm{g}}^{-}, v>0\right)$ and $\mathrm{O}\left({ }^{3} \mathrm{P}\right)$ photoproducts to the bath gas (described by eqn (7) and (8), respectively) govern the measured $C$ and $\theta$ (through eqn (6) and (3)). Importantly, eqn (7) and (8) predict these energy transfer timescales to depend inversely on the gas concentrations $\left[\mathrm{N}_{2}\right]$ and $\left[\mathrm{O}_{2}\right]$ that scale directly with $P_{0}$ through the ideal gas law. Therefore, from the outset, we expect these timescales to significantly increase at lower pressures and cause significant bias in PAS measurements of $C$ and increase the associated phase shift $\theta$.

In addition to the dependence of $k_{\mathrm{CF}}$ on $P_{0}$, the microphone response function also depends on the bath gas composition. This composition dependence was ignored in the last section, which considered measurements on aerosols suspended in air only. Changes in gas composition affect the microphone response function by impacting on various terms in eqn (12), including on the gas density (through changes in the mean molecular weight) and speed of sound. The dependence of $\rho$ on $P_{0}$ and the mean molecular weight $M$ for an ideal gas is given by:

$$
\rho=\frac{M}{R T} P_{0}
$$

in which $R$ is the molar gas constant, and $T$ is the temperature. Similarly, the speed of sound for an ideal gas is:

$$
v=\sqrt{\frac{\sigma R T}{M}}
$$

in which $\sigma$ is the adiabatic coefficient. Therefore, we can define the correction factor $k_{\mathrm{CF}}$ as the ratio of $k_{\mathrm{M}}$ at a given static pressure and composition to that at a reference pressure $\left(P_{0}=1000 \mathrm{hPa}\right)$ and composition (pure $\left.\mathrm{O}_{2}\right)$ :

$$
k_{\mathrm{CF}}=\frac{10^{3} S_{\mathrm{spk}} Q_{n}^{\ominus}}{P_{0} S_{\mathrm{spk}}^{\ominus} Q_{n}} \cdot \sqrt{\frac{M_{\mathrm{O}_{2}}}{y_{\mathrm{O}_{2}} M_{\mathrm{O}_{2}}+\left(1-y_{\mathrm{O}_{2}}\right) M_{\mathrm{N}_{2}}}}
$$

in which $M_{\mathrm{O}_{2}}$ and $M_{\mathrm{N}_{2}}$ are the molecular weights of $\mathrm{O}_{2}$ and $\mathrm{N}_{2}$, respectively, and $y_{\mathrm{O}_{2}}$ is the mole fraction of $\mathrm{O}_{2}$ in the bath gas. In deriving eqn (16), we make the same assumptions described earlier in deriving eqn (13), and also assume $\sigma$ is invariant with $P_{0}$ and composition; $\sigma$ varies by $<0.2 \%$ over our pressure range and for all mixing ratios of $\mathrm{O}_{2}$ and $\mathrm{N}_{2}$. To correct the measured 

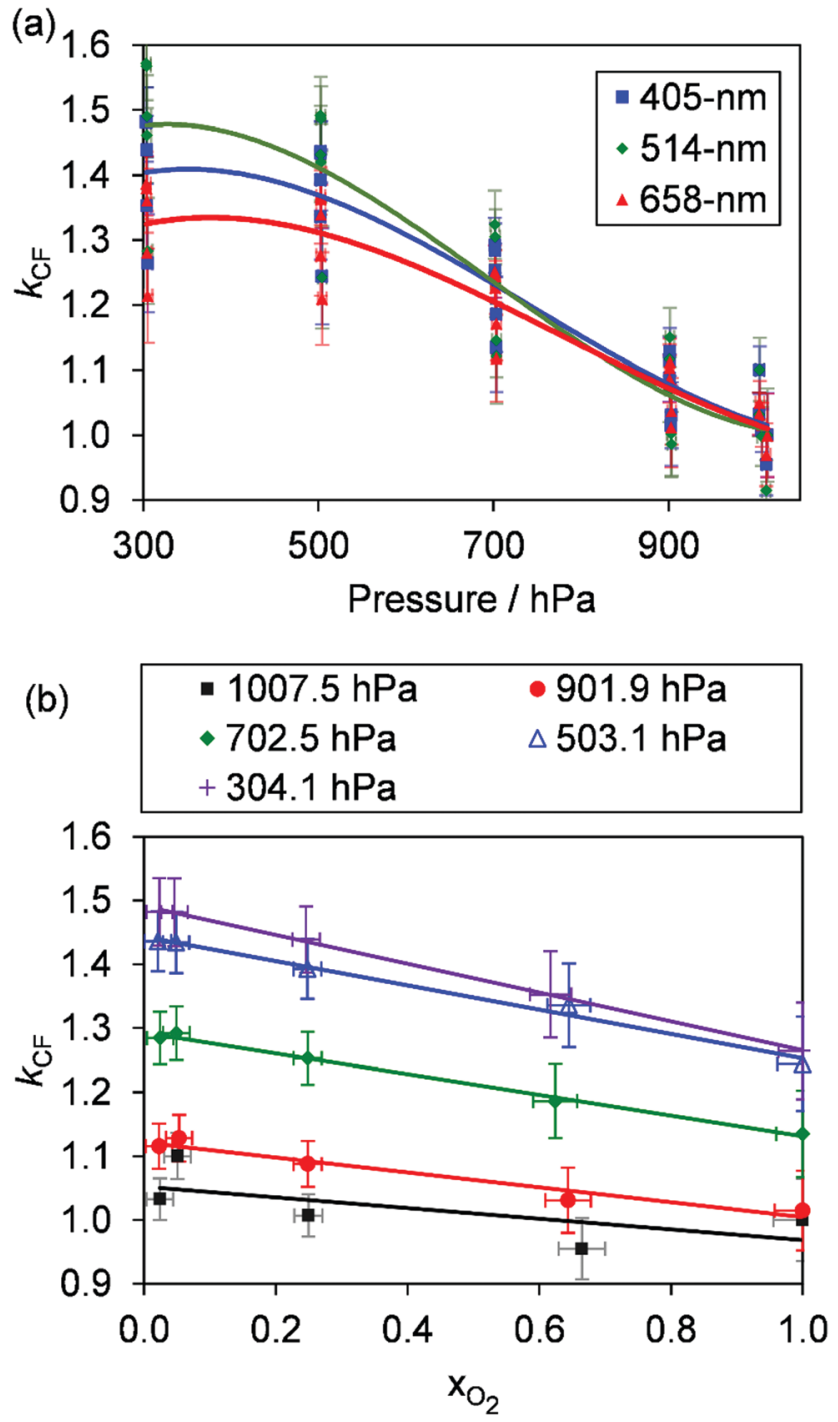

Fig. 3 (a) The variation in $k_{\mathrm{CF}}$ with $P_{0}$ for our three PAS spectrometers operating at wavelengths of 405,514 and $658 \mathrm{~nm}$. These data show larger variation at any given $P_{0}$ compared to those presented in Fig. 2(b) owing to the dependence of $k_{C F}$ on the bath gas composition. (b) The variation in $k_{\mathrm{CF}}$ with $x_{\mathrm{O}_{2}}$ at different $P_{0}$ for the $405 \mathrm{~nm}$ PAS instrument only. Lines are to guide the eye only.

PAS sensitivity $C$ for the pressure and composition dependent microphone sensitivity, we calculate the corrected sensitivity $C_{\text {cor: }}$ :

$$
C_{\text {cor }}=\frac{C}{k_{\mathrm{CF}}}
$$

Fig. 3(a) shows the pressure variation in $k_{\mathrm{CF}}$ for all the measurements of $C_{\text {cor }}$ presented in this section, i.e. for all bath gas compositions and pressures. While the general trend in $k_{\mathrm{CF}}$ with pressure is near-identical to that presented in Fig. 2, the $k_{\mathrm{CF}}$ for a given $P_{0}$ and instrument demonstrate higher variability caused by bath gas composition dependency. To demonstrate this latter effect, Fig. 3(b) shows the dependence of $k_{\mathrm{CF}}$ on $x_{\mathrm{O}_{2}}$ (the bath gas $\mathrm{O}_{2}$ mass fraction) for the $405 \mathrm{~nm}$ PAS instrument, with different data series corresponding to different $P_{0}$. For any fixed pressure within the range $300-1000 \mathrm{hPa}, k_{\mathrm{CF}}$ can vary by up to $\sim 15 \%$ as $x_{\mathrm{O}_{2}}$ increases from 0.0 to 1.0, with the microphones more sensitive in $\mathrm{N}_{2}$ compared to $\mathrm{O}_{2}$. The increased microphone response in $\mathrm{N}_{2}$ is expected; the lower molecular weight and density of $\mathrm{N}_{2}$ compared to that of $\mathrm{O}_{2}$ provides reduced damping of the microphone diaphragm motion.

Fig. 4 shows the variation in $C_{\text {cor }}$ with $x_{\mathrm{O} 2}$ for different $P_{0}$. Data are shown for measurements at $\lambda=405,658 \mathrm{~nm}$ only; measurement of $C_{\text {cor }}$ require PAS measurements of photoacoustic response in addition to CRDS measurements of the $\mathrm{O}_{3}$ absorption coefficient (see eqn (4)), and a CRDS spectrometer was not available at $\lambda=514 \mathrm{~nm}$. For both the 405 and $658 \mathrm{~nm}$ measurements, $C_{\text {cor }}$ is maximised as $x_{\mathrm{O} 2} \rightarrow 1$. This conclusion is different to our previous work that found strong wavelength dependence to the PAS sensitivity; while our previous $\lambda=658 \mathrm{~nm}$ measurements observed similar trends with a plateau in the PAS sensitivity attained for $x_{\mathrm{O} 2}>0.25$, measurements at $\lambda=405 \mathrm{~nm}$ demonstrated a maximum at $x_{\mathrm{O} 2} \sim 0.25$ before the sensitivity dropped as $x_{\mathrm{O} 2}$ increased further. ${ }^{24}$ We emphasise, however, that our previous work studied variations

\begin{tabular}{|lll|}
\hline$\cdot 1007.5 \mathrm{hPa}$ & $\bullet 901.9 \mathrm{hPa}$ & $\Delta 702.5 \mathrm{hPa}$ \\
$\cdot 503.1 \mathrm{hPa}$ & $\square 304.1 \mathrm{hPa}$ & \\
\hline
\end{tabular}
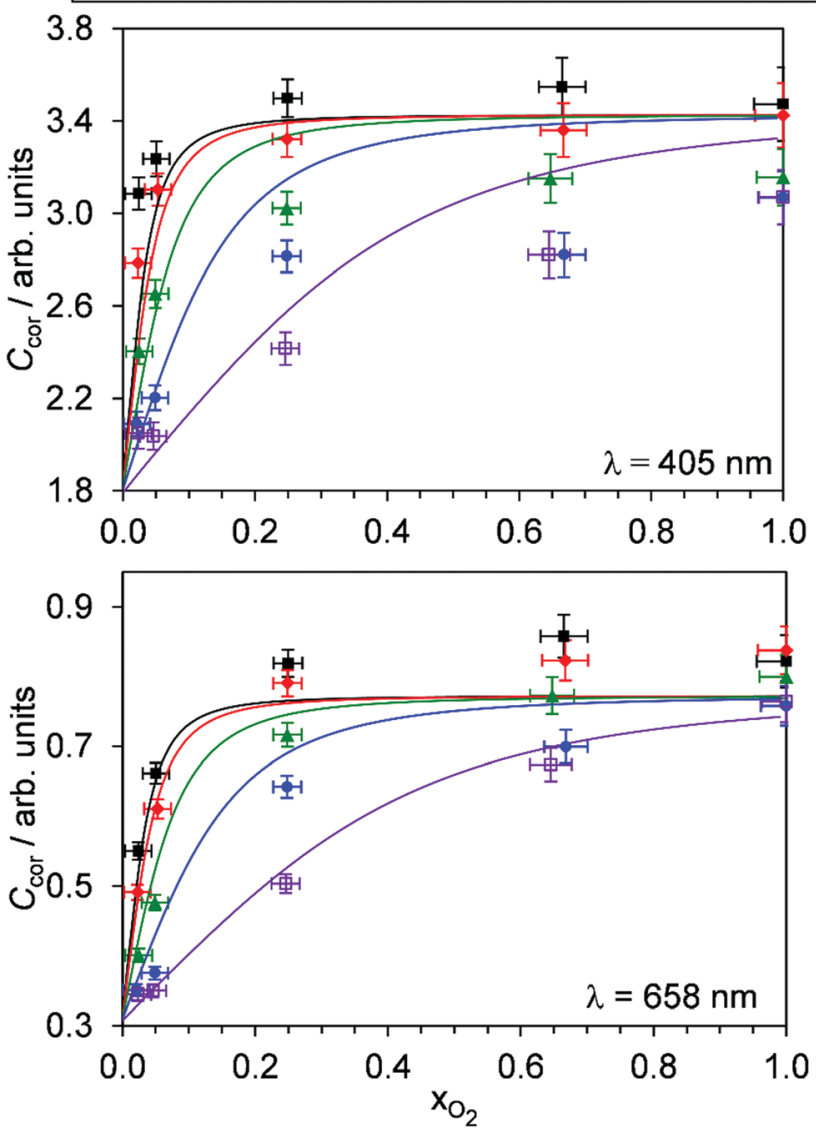

Fig. 4 The variation in $\mathrm{C}_{\text {cor }}$ with $\mathrm{O}_{2}$ mass fraction. The different data series correspond to different $P_{0}$ values. Data are shown for spectroscopy wavelengths of 405 and $658 \mathrm{~nm}$ only. Error bars represent $1 \sigma$ standard error in the measured quantities. The solid lines represent the best fit of the $\mathrm{O}_{3}$ photochemical relaxation model described by eqn (6) and (18)-(24) to measurements of both $C_{\text {cor }}$ and $\theta$. 
in $C$ and not $C_{\text {cor }}$, and thus did not explore corrections for bath gas mixing state. Indeed, Fig. S4-1 in the ESI $\dagger$ shows the PAS sensitivity without the correction for $k_{\mathrm{CF}}$ and demonstrates similar trends (for the $P_{0}=1007.5 \mathrm{hPa}$ data) to that in our previous publication. Moreover, the uncorrected $C$ show trends that are inconsistent with our expectation that $C$ should decrease for reduced $P_{0}$. For example, the $C$ for $405 \mathrm{~nm}$ measurements at $x_{\mathrm{O} 2}=0.25$ increases as $P_{0}$ decreases from 1007.5 hPa to $503.1 \mathrm{hPa}$, before a large reduction in $C$ as $P_{0}$ decreases further to a level of $304.1 \mathrm{hPa}$. Meanwhile, $C_{\text {cor }}$ in Fig. 4 shows variations with $P_{0}$ that are consistent with our understanding of the dependence of energy quenching on pressure (although we defer an explanation of our model fits in this figure to the following section).

For each of our three PAS spectroscopy wavelengths, Fig. 5 shows the variation in $\theta$ with $x_{\mathrm{O}_{2}}$ and $P_{0}$. Fig. S4-2 of the ESI $\dagger$ compares the measured $\theta$ from each spectrometer directly and shows that the phase shift variations with $P_{0}$ and $x_{\mathrm{O}_{2}}$ are similar for each measurement wavelength; all phase shift measurements are well correlated, with those measured at 514 and $658 \mathrm{~nm}$ wavelengths $\sim 15 \%$ larger than those recorded at $405 \mathrm{~nm}$. This similarity across wavelengths implies that the relaxation timescales of $\mathrm{O}\left({ }^{3} \mathrm{P}\right)$ and $\mathrm{O}_{2}\left(\mathrm{X}^{3} \Sigma_{\mathrm{g}}^{-}, v>0\right)$ are nearly independent of photolysis wavelength in the Chappuis band. To understand the variations in $C_{\text {cor }}$ and $\theta$ with $P_{0}$ and $x_{\mathrm{O}_{2}}$, we fit our photochemical model to our measurements.

4.2.1 Modelling the observed variations in $C$ and $\theta$ with pressure and bath gas composition. To model our measured $C_{\text {cor }}$, we fit eqn (6) to our measurements, using speakerexcitation measurements of the eigenmode frequency for each PAS instrument to calculate $\omega_{n}$, while the parameters $C_{0, \mathrm{O}}$ and $C_{0, \mathrm{O}_{2}^{*}}$ were varied as fit parameters. The timescales $\tau_{\mathrm{O}_{2}^{*}}$ and $\tau_{\mathrm{O}}$ are described by eqn (7) and (8), respectively, in terms of the bath gas concentrations $\left[\mathrm{O}_{2}\right]$ and $\left[\mathrm{N}_{2}\right]$. We can re-define these expressions as functions of $P_{0}$ and $x_{\mathrm{O} 2}$ :

$$
\begin{array}{r}
\tau_{\mathrm{O}_{2}^{*}}=\left(k_{\mathrm{O}_{2}^{*}-\mathrm{N}_{2}}\left(1-x_{\mathrm{O}_{2}}\right)\left(\frac{P_{0}}{1000 \mathrm{hPa}}\right)\right. \\
\left.+k_{\mathrm{O}_{2}^{*}-\mathrm{O}_{2}} x_{\mathrm{O}_{2}}\left(\frac{P_{0}}{1000 \mathrm{hPa}}\right)\right)^{-1} \\
\tau_{\mathrm{O}}=\left(k_{\mathrm{O}_{-} \mathrm{O}_{2}-\mathrm{N}_{2}} x_{\mathrm{O}_{2}}\left(1-x_{\mathrm{O}_{2}}\right)\left(\frac{P_{0}}{1000 \mathrm{hPa}}\right)^{2}\right. \\
\left.+k_{\mathrm{O}_{-} \mathrm{O}_{2}-\mathrm{O}_{2}}\left(\frac{x_{\mathrm{O}_{2}} P_{0}}{1000 \mathrm{hPa}}\right)^{2}\right)^{-1}
\end{array}
$$

To model the phase shift data, we recognise that the microphone waveform will consist of contributions from the relaxation of both $\mathrm{O}\left({ }^{3} \mathrm{P}\right)$ and $\mathrm{O}_{2}\left(\mathrm{X}^{3} \Sigma_{\mathrm{g}}^{-}, v>0\right)$ photoproducts. While one photoproduct might relax on a long timescale (i.e. have a large associated photoacoustic phase shift), longer relaxation timescales will produce an associated lower contribution to the overall photoacoustic response (see eqn (5)) and the measured $\theta$ from the microphone waveform will be a photoacoustic

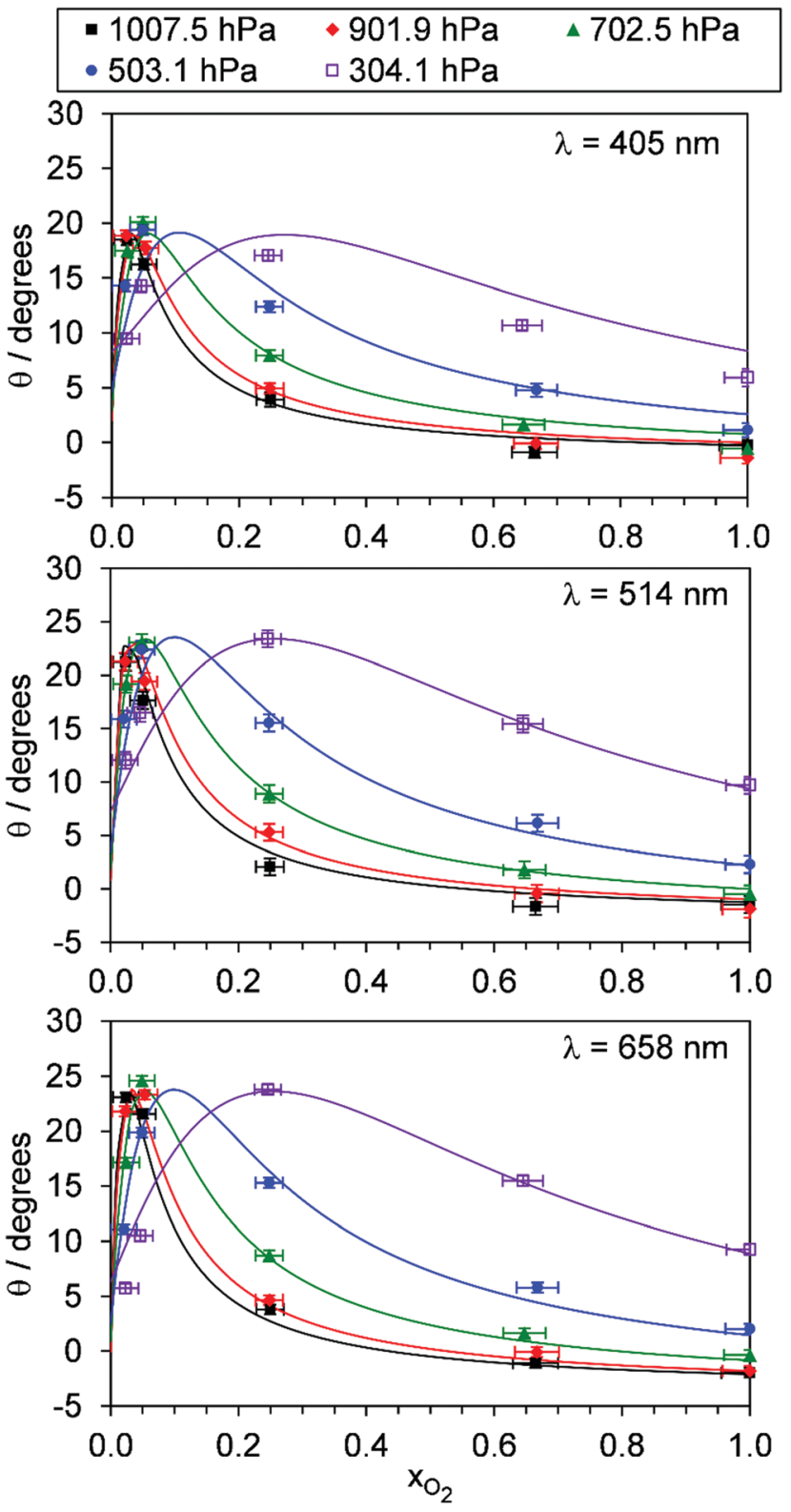

Fig. 5 The variation in photoacoustic phase shift with $x_{\mathrm{O}_{2}}$. The different data series correspond to different $P_{0}$ values. Data are shown for spectroscopy wavelengths of 405, 514 and $658 \mathrm{~nm}$. Error bars represent $1 \sigma$ standard error in the measured quantities. The solid lines represent the best fit of the $\mathrm{O}_{3}$ photochemical relaxation model described by eqn (6) and (18)-(24) to measurements of both $C_{\text {cor }}$ and $\theta$.

amplitude weighting of the individual phase shift contributions from relaxation of both the nascent photoproducts. Thus, the measured $\theta$ consists of contributions from the relaxation of both $\mathrm{O}\left({ }^{3} \mathrm{P}\right)$ and $\mathrm{O}_{2}\left(\mathrm{X}^{3} \Sigma_{\mathrm{g}}^{-}, v>0\right)$ photoproducts weighted by their respective photoacoustic amplitude contributions $C_{\mathrm{O}}$ and $C_{\mathrm{O}_{2}^{*}}$ to $C_{\text {cor: }}$ :

$$
C_{\mathrm{O}}=\frac{C_{0, \mathrm{O}}}{\sqrt{1+\left(\omega_{n} \tau_{\mathrm{O}}\right)^{2}}}
$$




$$
\begin{gathered}
C_{\mathrm{O}_{2}^{*}}=\frac{C_{0, \mathrm{O}_{2}^{*}}}{\sqrt{1+\left(\omega_{n} \tau_{\mathrm{O}_{2}^{*}}\right)^{2}}} \\
\theta=\frac{C_{\mathrm{O}} \theta_{\mathrm{O}}+C_{\mathrm{O}_{2}^{*}} \theta_{\mathrm{O}_{2}^{*}}}{C_{\mathrm{O}}+C_{\mathrm{O}_{2}^{*}}}
\end{gathered}
$$

in which $\theta_{\mathrm{O}}$ and $\theta_{\mathrm{O}_{2}^{*}}$ are the phase shifts attributed to $\mathrm{O}\left({ }^{3} \mathrm{P}\right)$ and $\mathrm{O}_{2}\left(\mathrm{X}^{3} \Sigma_{\mathrm{g}}^{-}, v>0\right)$ and depend on their associated quenching timescales via eqn (3):

$$
\begin{gathered}
\theta_{\mathrm{O}}=\tan ^{-1}\left(\omega_{n} \tau_{\mathrm{O}}\right) \\
\theta_{\mathrm{O}_{2}^{*}}=\tan ^{-1}\left(\omega_{n} \tau_{\mathrm{O}_{2}^{*}}\right)
\end{gathered}
$$

Thus, we performed a non-linear least-squares optimisation of our photochemical model described by eqn (6) and (18)-(24) to our measured $C_{\text {cor }}$ and $\theta$, varying the parameters $C_{0, \mathrm{O}}, C_{0, \mathrm{O}_{2}^{*}}$, $k_{\mathrm{O}_{2}^{*}-\mathrm{N}_{2}}, \quad k_{\mathrm{O}_{2}^{*}-\mathrm{O}_{2}}, \quad k_{\mathrm{O}-\mathrm{O} 2-\mathrm{N} 2}$ and $k_{\mathrm{O}-\mathrm{O} 2-\mathrm{O} 2}$. This least-squares minimisation optimised our model simultaneously to measured $C_{\text {cor }}$ and $\theta$ for all measurement wavelengths. In this least-squares fit, we constrained $k_{\mathrm{O}_{2}^{*}-\mathrm{N}_{2}}, k_{\mathrm{O}_{2}^{*}-\mathrm{O}_{2}}, k_{\mathrm{O}-\mathrm{O} 2-\mathrm{N} 2}$ and $k_{\mathrm{O}-\mathrm{O} 2-\mathrm{O} 2}$ to be invariant with photolysis wavelength, justified by our aforementioned observations of only a weak dependence of phase shift on wavelength. Moreover, this latter treatment of rate constants is consistent with that in our previous work. ${ }^{24}$ Similarly, we followed the same treatment in our previous work by allowing $C_{0, \mathrm{O}}$ and $C_{0, \mathrm{O}_{2}^{*}}$ to vary with wavelength. In addition to these parameters, we allowed a small phase shift offset (with magnitudes up to $3.4^{\circ}$ ) to be added to the modelled phase at each photolysis wavelength to account for inaccuracies in the subtraction of instrument phase shifts. The best fit parameters from this least-squares optimisation are summarised in Table 1 and the modelled $C_{\text {cor }}$ and $\theta$ are compared against measurements in Fig. 4 and 5, respectively.

Fig. 4 shows that, overall, the model is in good agreement with the measured trends in $C_{\text {cor }}$ with both $P_{0}$ and $x_{\mathrm{O} 2}$. On average, the mean absolute difference between the modelled and measured $C_{\text {cor }}$ is $7.3 \%$ and $5.1 \%$ at wavelengths of 405 and $658 \mathrm{~nm}$, respectively. There are numerous sources that could contribute to these small levels of disagreement. First, the error bars on Fig. 4 for the measured $C_{\text {cor }}$ reflect the $1 \sigma$ precision and do not include uncertainty arising from potential biases in the correction factor $k_{\mathrm{CF}}$. Our measurements in Section 4.1 indicated that $k_{\mathrm{CF}}$ is accurate to within $5 \%$ for $P_{0}$ over the range 300-1000 hPa. However, these accuracy assessments apply to a bath gas of air only, and the accuracy in our $k_{\mathrm{CF}}$ characterisations for different bath gas compositions has not been assessed. Second, our model fit could be optimised to a local minimum. The high number of fit parameters (13), that were varied in fitting our photochemical model to measurements, increases the likelihood of optimising to a local minimum in the least-squares residual. We mitigated against optimisation to a local minimum by initialising our fit parameters from numerous initial values prior to minimisation, and these best fit parameters converged consistently to the same optimal values. Third, our quenching model contains some deficiency
Table 1 Parameters describing the best fit of the photochemical relaxation model (described in the main text) to measured $C_{\text {cor }}$ and $\theta$ values

\begin{tabular}{llll}
\hline PAS wavelength/nm & 405 & 514 & 658 \\
\hline$C_{0, \mathrm{O}}$ & 1.61 & 1.86 & 0.46 \\
$C_{0, \mathrm{O}_{2}^{*}}$ & 1.82 & 1.37 & 0.31 \\
$k_{\mathrm{O}-\mathrm{O}_{2}-\mathrm{N}_{2}} / \mathrm{bar}^{-2} \mathrm{~s}^{-1}$ & $2.18 \times 10^{5}$ & & \\
$k_{\mathrm{O}-\mathrm{O}_{2}-\mathrm{O}_{2}} / \mathrm{bar}^{-2} \mathrm{~s}^{-1}$ & $2.77 \times 10^{5}$ & & \\
$k_{\mathrm{O}_{2}^{*}-\mathrm{N}_{2}} / \mathrm{bar}^{-1} \mathrm{~s}^{-1}$ & $1.97 \times 10^{5}$ & & \\
$k_{\mathrm{O}_{2}^{*}-\mathrm{O}_{2}} / \mathrm{bar}^{-1} \mathrm{~s}^{-1}$ & $2.19 \times 10^{6}$ & & \\
Offset in $\theta /^{\circ}$ & -1.3 & -2.5 & -3.4 \\
$C_{0, \mathrm{O}_{2}^{*}} / C_{0, \mathrm{O}}$ & 1.13 & 0.74 & 0.68 \\
$k_{\mathrm{O}-\mathrm{O}_{2}-\mathrm{O}_{2}} / k_{\mathrm{O}-\mathrm{O}_{2}-\mathrm{N}_{2}}$ & 1.27 & & \\
$k_{\mathrm{O}_{2}^{*}-\mathrm{O}_{2}} / k_{\mathrm{O}_{2}^{*}-\mathrm{N}_{2}}$ & 11.1 & & \\
\end{tabular}

in modelling accurately the quenching of energy from $\mathrm{O}\left({ }^{3} \mathrm{P}\right)$. Specifically, we highlight that eqn (19) for the timescale $\tau_{\mathrm{O}}$ represents the timescale with which nascent $O\left({ }^{3} \mathrm{P}\right)$ is lost through recombination with bath $\mathrm{O}_{2}\left(\mathrm{X}^{3} \Sigma_{\mathrm{g}}^{-}\right)$and the resulting unstable intermediate $\mathrm{O}_{3}^{\ddagger}(\tilde{\mathrm{X}})$ stabilised via a further collision with a bath gas species to form vibrationally hot $\mathrm{O}_{3}(\tilde{\mathrm{X}}, v>0)$ (see reactions (R3)-(R5)). The vibrational energy from this hot $\mathrm{O}_{3}(\tilde{\mathrm{X}}, v>0)$ product remains to be transferred to the bath gas. Thus, in stating $\tau_{\mathrm{O}}$ to represent the timescale of energy transfer from the $\mathrm{O}\left({ }^{3} \mathrm{P}\right)$ photoproduct to bath gas translational energy, we inherently assume that $\mathrm{V}-\mathrm{T}$ energy transfer from $\mathrm{O}_{3}(\tilde{\mathrm{X}}, v>0)$ to the bath gas is fast compared to the photoacoustic modulation period..$^{24}$ Indeed, Ménard-Bourcin et al. ${ }^{53}$ and Zeninari et al. ${ }^{54}$ measured similar rates for the single-quantum $\mathrm{V}-\mathrm{T}$ energy transfer from vibrationally excited $\mathrm{O}_{3}(\tilde{\mathrm{X}}, v>0)$ to bath $\mathrm{O}_{2}$ or $\mathrm{N}_{2}$ of $\sim 7.6 \times 10^{5} \mathrm{~s}^{-1} \mathrm{~atm}^{-1}$, corresponding to a characteristic timescale of $1.4 \mu \mathrm{s}$ at $P_{0}=950 \mathrm{hPa}$. This timescale is fast compared to the 620-660 $\mu$ s modulation timescales for our PAS instruments. However, $\mathrm{O}_{3}(\tilde{\mathrm{X}}, v>0)$ could be formed in vibrational states with populations as high as $(7,0,0)$ at short photolysis energies ${ }^{55}$ and, at reduced pressures, the timescale for quenching of this state could be up to $\sim 60 \mu \mathrm{s}$ for $P_{0}=300 \mathrm{hPa}$. Fig. 6 shows our modelled timescales $\tau_{\mathrm{O}}$ and $\tau_{\mathrm{O} 2}$ with variation in $x_{\mathrm{O} 2}$ and $P_{0}$. Clearly, an upper estimate of $60 \mu \mathrm{s}$ for the V-T energy transfer from $\mathrm{O}_{3}(\tilde{\mathrm{X}}, v>0)$ is comparable to the modelled timescale for $\tau_{\mathrm{O}}$. However, the addition of the $\mathrm{O}_{3}(\tilde{\mathrm{X}}, v>0) \mathrm{V}-\mathrm{T}$ energy transfer step is not straightforward and the additional fit parameters that arise reduce the fidelity of our best-fit model. Moreover, we expect the mean timescale for $\mathrm{V}-\mathrm{T}$ relaxation of $\mathrm{O}_{3}(\tilde{\mathrm{X}}, v>0)$ to be much less than this $60 \mu \mathrm{s}$ upper estimate, as an ensemble of $\left(v_{1}, v_{2}, v_{3}\right)$ states is produced and the single quantum transition from some states (namely, the $v_{2}$ state) is particularly fast to the ground state.

Fig. 5 shows good agreement between our modelled and measured phase shifts; the mean discrepancies between these are $1.3^{\circ}, 1.1^{\circ}$ and $1.0^{\circ}$ at our 405,514 and $658 \mathrm{~nm}$ spectroscopy wavelengths, respectively. Importantly, the measured values are subject to less uncertainty compared to those for $C_{\text {cor }}$, as the measurement of $\theta$ does not require normalisation for $\mathrm{O}_{3}$ concentrations using independent (e.g. CRDS) techniques or a correction for the microphone response function. We also 

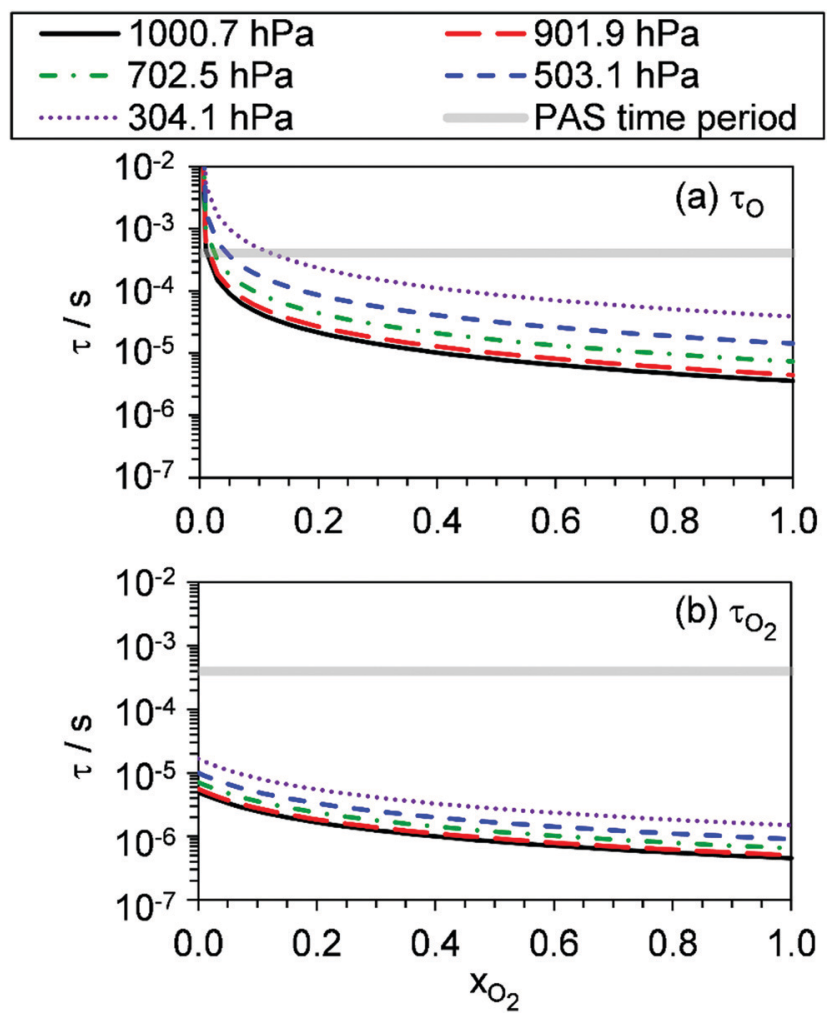

Fig. 6 The best-fit modelled timescales (a) $\tau_{\mathrm{O}}$, and (b) $\tau_{\mathrm{O}_{2}}$, with variation in $x_{\mathrm{O}_{2}}$. The different data series correspond to different $P_{0}$ values. For reference, the grey shaded area represents the 620-660 $\mu$ s laser power modulation time periods for the PAS instruments used in this work.

stress that phase shift is a direct measure of relaxation timescales, and the level of agreement between our measurements and our best-fit photochemical model indicates that the aforementioned model assumption of instantaneous $\mathrm{V}-\mathrm{T}$ relaxation of $\mathrm{O}_{3}(\tilde{\mathrm{X}}, v>0)$ is reasonable. The measured $\theta$ might be expected to follow a similar trend to that of the best-fit model timescales presented in Fig. 6, with large values at low $x_{\mathrm{O}_{2}}$ and $P_{0}$, which then decrease with increasing $x_{\mathrm{O}_{2}}$ and $P_{0}$. This trend in $\theta$ is largely observed in the $x_{\mathrm{O}_{2}}$ range $\sim 0.2-1.0$, while $\theta$ approaches zero as $x_{\mathrm{O}_{2}} \rightarrow 0$. To understand this latter observation, we recall that the $\theta$ derived from the microphone waveform corresponds to a photoacoustic amplitude weighting of the phase shifts associated with energy transfer from both nascent photoproducts $\mathrm{O}\left({ }^{3} \mathrm{P}\right)$ and $\mathrm{O}_{2}\left(\mathrm{X}^{3} \Sigma_{\mathrm{g}}^{-}, v>0\right)$. Eqn (19) shows that the timescale for energy transfer from $\mathrm{O}\left({ }^{3} \mathrm{P}\right)$ tends to infinity as $x_{\mathrm{O}_{2}} \rightarrow 0$. This long timescale is associated with a large $90^{\circ}$ phase for $\theta_{\mathrm{O}}$ (eqn (23)). However, the associated contribution to the photoacoustic signal is very small; Fig. 4 demonstrates a significant drop in $C_{\text {cor }}$ as $x_{\mathrm{O}_{2}} \rightarrow 0$. Thus, recalling that the measured $\theta$ is a photoacoustic amplitude weighting of the two phase shift contributions from quenching of $\mathrm{O}\left({ }^{3} \mathrm{P}\right)$ and $\mathrm{O}_{2}\left(\mathrm{X}^{3} \Sigma_{g}^{-}, v>0\right)$ (eqn (22)), the observed phase shift is expected to tend to zero as $x_{\mathrm{O}_{2}} \rightarrow 0$.

Our measurements allow us to form recommendations on using $\mathrm{O}_{3}$-laden gas as a calibrant of photoacoustic spectrometers. Many researchers calibrate using $\mathrm{O}_{3}$-laden air. ${ }^{10,15,22,40}$
Our $C_{\text {cor }}$ measurements demonstrate that such calibrations in air (i.e. $x_{\mathrm{O}_{2}} \sim 0.25$ ) are accurate at $P_{0}$ in excess of $1000 \mathrm{hPa}$, and this conclusion is reinforced by our measured $\theta$ that are $<5^{\circ}$ and indicate fast energy transfer, compared to our photoacoustic modulation period, from the Chappuis band photoproducts to $\mathrm{N}_{2}$ and $\mathrm{O}_{2}$ bath species. However, the accuracy of these calibrations is very sensitive to $P_{0}$, with notable biases in $C_{\text {cor }}$ at $P_{0}<900 \mathrm{hPa}$. In contrast, calibrations using $\mathrm{O}_{3}$-laden $\mathrm{O}_{2}$ exhibit no detectable bias for $P_{0}>900 \mathrm{hPa}$ and negligible phase shift for $P_{0}>500 \mathrm{hPa}$, with the sensitivity in $C_{\text {cor }}$ to $P_{0}$ much lower than that at other bath gas mixing states. For measurements at PAS modulation frequencies $<2 \mathrm{kHz}$, we thus recommend calibrating PAS instruments using $\mathrm{O}_{3}$-laden gas in a bath gas with $x_{\mathrm{O}_{2}} \geq 0.25$ and $P_{0}>900 \mathrm{hPa}$. The latter pressure threshold can be relaxed at higher $x_{\mathrm{O}_{2}}$. Crucially, these criteria depend strongly on the photoacoustic modulation timescale; some researchers may wish to perform measurements at very high frequencies ( $>30 \mathrm{kHz}$ ) such as that afforded by tuning fork detection. Such researchers will find the timescales presented in Fig. 6 most useful in ascertaining whether such calibrations are accurate given their choice of photoacoustic modulation timescale, $x_{\mathrm{O}_{2}}$, and $P_{0}$.

4.2.2 Understanding the best-fit model rate constants. In this final discussion section, we rationalise our best-fit model rate constants. First, we highlight that the ratio $C_{0, \mathrm{O}_{2}^{*}} / C_{0, \mathrm{O}}$ increases with photolysis energy, consistent with an increasing fraction of energy partitioning to $\mathrm{O}_{2}(\mathrm{X})$ vibrational modes at shorter wavelengths. This finding is consistent with our previous measurements, ${ }^{24}$ and with studies reported by others that show that higher $\mathrm{O}_{2}(\mathrm{X})$ vibrational states are populated following photodissociation in the Chappuis band at shorter wavelengths. ${ }^{45,46}$

The quotient $k_{\mathrm{O}-\mathrm{O} 2-\mathrm{O} 2} / k_{\mathrm{O}-\mathrm{O} 2-\mathrm{N} 2}$ is 1.27 . Previously, we demonstrated that $k_{\mathrm{O}-\mathrm{O} 2-\mathrm{O} 2}$ and $k_{\mathrm{O}-\mathrm{O} 2-\mathrm{N} 2}$ are given by $k_{1} k_{3} / k_{2}$, in which $k_{1}, k_{2}$ and $k_{3}$ are defined in reactions (R3)-(R5). ${ }^{24}$ The rate coefficients $k_{1}$ and $k_{2}$ are independent of bath gas, while $k_{3}$ depends on the ability of the bath gas to quench energy efficiently from the unstable $\mathrm{O}_{3}^{ \pm}(\tilde{\mathrm{X}})$ intermediate. Therefore, the ratio $k_{\mathrm{O}-\mathrm{O} 2-\mathrm{O} 2} / k_{\mathrm{O}-\mathrm{O} 2-\mathrm{N} 2}$ is equivalent to $k_{3, \mathrm{O} 2} / k_{3, \mathrm{~N} 2}$. While $\mathrm{V}-\mathrm{T}$ rates for $\mathrm{O}_{3}^{\ddagger}(\tilde{\mathrm{X}})$ have not been studied directly, these rates have been studied for excitations of the $\left(v_{1}, v_{2}, v_{3}\right)$ states of the stable $\mathrm{O}_{3}(\tilde{\mathrm{X}}, v>0)$ species. $^{53,54}$ The direct relaxation from $v_{1}$ (symmetric stretch) and $v_{3}$ (asymmetric stretch) are comparatively slow compared to the interconversion $v_{1}, v_{3} \rightarrow v_{2}$ and then subsequent quenching from $v_{2}$. Thus, vibrationally excited $\mathrm{O}_{3}$ is quenched mostly from the $v_{2}$ state. The ratio of $v_{2}$ quenching rates in $\mathrm{O}_{2}$ and $\mathrm{N}_{2}$ bath gases is $1.10 \pm 0.17$ (Menard-Bourcin et al. $)^{53}$ or $1.07 \pm 0.16$ (Zeninari et al.), ${ }^{54}$ in near agreement with our measured ratio $k_{\mathrm{O}-\mathrm{O} 2-\mathrm{O} 2} / k_{\mathrm{O}-\mathrm{O} 2-\mathrm{N} 2}=$ 1.27. Our new measurements reconcile a discrepancy in our previously published work in which we determined $k_{\mathrm{O}-\mathrm{O} 2-\mathrm{O} 2} / k_{\mathrm{O}-\mathrm{O} 2-\mathrm{N} 2}=11.1$. This reconciliation is achieved through the additional constraint on the best-fit rate coefficients provided by phase shift measurements, and through the improved accuracy of our PAS sensitivity measurements; measured $C$ are now 
corrected for the microphone response function dependence on bath gas density. For the individual rate coefficients, we measure $k_{\mathrm{O}-\mathrm{O} 2-\mathrm{O} 2}=2.77 \times 10^{5} \mathrm{~s}^{-1}$ and $k_{\mathrm{O}-\mathrm{O} 2-\mathrm{N} 2}=2.18 \times 10^{5} \mathrm{~s}^{-1}$ at $P_{0}=1000 \mathrm{hPa}$, while Ménard-Bourcin et al. predict limiting rates for $v_{2}$ quenching to be faster at rates of $(8.23 \pm 0.8) \times$ $10^{5} \mathrm{~s}^{-1}$ in $\mathrm{O}_{2}$ and $(7.5 \pm 0.9) \times 10^{5} \mathrm{~s}^{-1}$ in $\mathrm{N}_{2} \cdot{ }^{53}$ Similarly, Zeninari et al. reported $v_{2}$ quenching rates of $(5.6 \pm 0.8) \times$ $10^{5} \mathrm{~s}^{-1}$ in $\mathrm{O}_{2}$ and $(5.3 \pm 0.4) \times 10^{5} \mathrm{~s}^{-1}$ in $\mathrm{N}_{2} \cdot{ }^{54}$ However, there are several factors that contribute to an expected difference between our rates and the limiting $v_{2}$ quenching rates reported in previous studies. First, the total relaxation rates $k_{\mathrm{O}-\mathrm{O} 2-\mathrm{O} 2}$ and $k_{\mathrm{O}-\mathrm{O} 2-\mathrm{N} 2}$ are attributed to relaxation through the transition $v_{1}$, $v_{3} \rightarrow v_{2}$ in addition to that from $v_{2}$. Second, the total quenching rate will depend on the number of quanta in vibrational energy levels, with the rates reported in previous studies corresponding to single quantum transitions, while our measurements will involve $\mathrm{O}_{3}$ with multiple vibrational quanta distributed over $v_{1}$, $v_{2}$, and $v_{3}$. Third, our measured rates correspond to quenching from vibrational states above the dissociation energy threshold for $\mathrm{O}_{3}$, while the rates reported by the aforementioned literature studies correspond to vibrational states below this threshold.

The quotient $k_{\mathrm{O}_{2}^{*}-\mathrm{O}_{2}} / k_{\mathrm{O}_{2}^{*}-\mathrm{N}_{2}}$ is 11.1. In previous work, we reported $k_{\mathrm{O}_{2}^{*}-\mathrm{O}_{2}} / k_{\mathrm{O}_{2}^{*}-\mathrm{N}_{2}}=0.6,{ }^{24}$ although we highlighted that the fit of our photochemical model was rather insensitive to changes in this ratio when fitting to PAS sensitivity measurements. However, the phase data that are now included in our model optimisation enable more sensitive retrievals of $k_{\mathrm{O}_{2}^{*}-\mathrm{O}_{2}}$ and $k_{\mathrm{O}_{2}^{*}-\mathrm{N}_{2}}$. The best fit model rate constant $k_{\mathrm{O}_{2}^{*}-\mathrm{O}_{2}}=2.19 \times$ $10^{6} \mathrm{bar}^{-1} \mathrm{~s}^{-1}\left(8.85 \times 10^{-14} \mathrm{~cm}^{3} \mathrm{~s}^{-1}\right)$ corresponds to a relaxation timescale of $\sim 0.5 \mu \mathrm{s}$ for $x_{\mathrm{O}_{2}} \rightarrow 1$ and for $P_{0}=1000 \mathrm{hPa}$ (see Fig. 6(b)), while the associated measured phase shifts $<3^{\circ}$ correspond directly to quenching timescales $<5 \mu \mathrm{s}$. These timescales indicate that $\mathrm{O}_{2}(\mathrm{X}, v>0)$ relaxation occurs efficiently relative to our PAS modulation periods as $x_{\mathrm{O}_{2}} \rightarrow 1$ and for $P_{0}>1000 \mathrm{hPa}$. We can compare our measured rates of $\mathrm{O}_{2}(\mathrm{X}, v>0)$ quenching with theoretical $\mathrm{V}-\mathrm{T}$ rates calculated by Coletti and Billing, who predicted rates $<2 \times 10^{-17} \mathrm{~cm}^{3} \mathrm{~s}^{-1}$ for vibrational quantum numbers $v<10$ at ambient temperature. ${ }^{56}$ Meanwhile, Huestis performed a reanalysis of a range of $\mathrm{V}-\mathrm{T}$ energy transfer studies of vibrationally excited $\mathrm{O}_{2}$ and recommended a $\mathrm{V}-\mathrm{T}$ quenching rate of $2.7 \times 10^{-18} \mathrm{~cm}^{3} \mathrm{~s}^{-1}$ for $v=1$ at temperatures of $300 \mathrm{~K}^{57}$ These rates are $\sim 4$ orders of magnitude slower than the rates we determine for $k_{\mathrm{O}_{2}^{*}-\mathrm{O}_{2}}$ as $x_{\mathrm{O}_{2}} \rightarrow 1$ and for $P_{0}>1000 \mathrm{hPa}$. Such slow $\mathrm{V}-\mathrm{T}$ rates would correspond to phase shifts approaching $90^{\circ}$ at our PAS modulation frequencies. Although the stated uncertainties in the $\mathrm{V}-$ $\mathrm{T}$ rates reported by Coletti and Billing (2002) are $>25 \%$ for $v<$ 10 , we fail to reconcile our measurements with predictions from theoretical models. We cannot find any literature measurements to support the $\mathrm{V}-\mathrm{T}$ rates reported by theoretical studies for $v<8$; Park and Slanger ${ }^{58}$ and Hickson et al. ${ }^{59}$ only confirmed the predicted total quenching rates (the sum of $\mathrm{V}-\mathrm{T}$ and vibrational-to-vibrational energy transfer rates) for $v>8$. Nonetheless, it is unlikely that we can explain our observed
$\mathrm{O}_{2}(\mathrm{X}, v>0)$ quenching rate by considering quenching by $\mathrm{O}_{2}$ bath gas alone. To reconcile our measurements with models, it is possible that $\mathrm{O}_{2}(\mathrm{X}, v>0)$ is effectively quenched by $\mathrm{O}_{3}(\tilde{\mathrm{X}})$ in the bath gas. The reverse energy transfer process (i.e. $\mathrm{O}_{3}(\tilde{\mathrm{X}}, v>0)$ quenched by $\left.\mathrm{O}_{2}(\mathrm{X})\right)$ has been studied extensively and the corresponding $\mathrm{V}-\mathrm{T}$ rate is $\sim 3 \times 10^{-14} \mathrm{~cm}^{3} \mathrm{~s}^{-1} \cdot{ }^{53,54}$ However, the concentrations of $\mathrm{O}_{3}$ measured by our CRDS instruments are $<2000 \mathrm{ppm}$, which will reduce the total rate of $\mathrm{O}_{2}$ quenching by $\mathrm{O}_{3}$. Alternatively, there may be trace impurities in our bath gas, although this is unlikely given the use of high purity N5-grade $\mathrm{O}_{2}$. For the relaxation of $\mathrm{O}_{2}(\mathrm{X}, v>0)$ in bath gas $\mathrm{N}_{2}$, we determine $k_{\mathrm{O}_{2}^{*}-\mathrm{N}_{2}}=1.97 \times 10^{5} \mathrm{bar}^{-1} \mathrm{~s}^{-1}$. For this relaxation, Billing reported $\mathrm{V}-\mathrm{T}$ rate constants for $v>13$ only, ${ }^{60}$ with such high $v$ not expected to be populated at our photolysis energies; Flöthmann et al. ${ }^{45}$ and Levene et al. show that $v=0$ dominates at all wavelengths, ${ }^{46}$ with a shoulder in this distribution appearing corresponding to population of $v=4-8$ at $\lambda=450-500 \mathrm{~nm}$, and with higher $v$ expected to dominate for $\lambda<450 \mathrm{~nm}$. Thus, it is again difficult to assess the accuracy of our determined rate constant in the context of previous theoretical investigations.

\section{Conclusions}

We have demonstrated the accurate correction of photoacoustic response for changes in microphone response function $k_{\mathrm{M}}$ with static pressure $P_{0}$ from measurements of the relative variations in microphone amplitude and PAS instrument quality factors using a speaker transducer for PAS cell eigenmode excitation. Indeed, our correction strategy is similar to that reported by other researchers. ${ }^{7}$ This method of correcting for $k_{\mathrm{M}}$ has the advantage that regular and fast characterisations of the microphone response function (via speaker-excitation measurements of $Q_{n}$ and $S_{\mathrm{spk}}$ ) can be made without the need for controlled generation of a test sample. This microphone response calibration method enabled the first studies of the pressure dependence to photoacoustic sensitivity $\left(C_{\text {cor }}\right)$ for $\mathrm{O}_{3}$-laden gas samples in the Chappuis band $(\lambda \sim 400-700 \mathrm{~nm})$ for various bath gas mass mixtures containing mass fractions of $\mathrm{O}_{2}\left(x_{\mathrm{O}_{2}}\right)$ in $\mathrm{N}_{2}$. Moreover, we have reported associated measurements of the phase shift $\theta$ that allow direct assessments of photoproduct relaxation timescales. There are strong variations in both $C_{\text {cor }}$ and $\theta$ with $P_{0}$ and $x_{\mathrm{O}_{2}}$ that are described well by the best-fit of a photochemical relaxation model we develop that retrieves the associated variations in the timescales for energy transfer (see Fig. 6) from the nascent Chappuis band photoproducts $\left(\mathrm{O}\left({ }^{3} \mathrm{P}\right)\right.$ and $\left.\mathrm{O}_{2}(\mathrm{X}, v>0)\right)$ to translational motion of the bath gas species. We have shown that the accuracy of $\mathrm{O}_{3}$-based calibrations of visible-wavelength PAS instruments is optimal as $x_{\mathrm{O}_{2}} \rightarrow 1$, in agreement with the conclusions of studies by other researchers. ${ }^{23}$ The reduced $C_{\text {cor }}$ and extended timescales for photoproduct relation in $\mathrm{N}_{2}$ bath gas are associated with the inefficient recombination of $\mathrm{O}\left({ }^{3} \mathrm{P}\right)$ with $\mathrm{O}_{2}$ and the slower $\mathrm{V}-\mathrm{T}$ relation of $\mathrm{O}_{2}(\mathrm{X}, v>0)$ in collisions with $\mathrm{N}_{2}$. For measurements at PAS modulation 
frequencies $<2 \mathrm{kHz}$, we recommend that measurements in the Chappuis band are performed for $x_{\mathrm{O}_{2}} \geq 0.25$ and $P_{0}>900 \mathrm{hPa}$, although the latter pressure threshold can be relaxed at higher $x_{\mathrm{O}_{2}}$. Crucially, these criteria depend strongly on the photoacoustic modulation timescale and researchers wishing to assess the accuracy of relevant measurements at higher PAS modulation frequencies should find the timescales presented in Fig. 6 useful.

\section{Conflicts of interest}

There are no conflicts to declare.

\section{Acknowledgements}

The Met Office funded the development of laboratory instruments for this work. Michael I. Cotterell acknowledges the support of the Natural Environment Research Council through an Independent Research Fellowship (NE/S014314/1). Jim M. Haywood and Michael I. Cotterell thank the Natural Environment Research Council for support through the CLARIFY-2017 grant (NE/L013797/1).

\section{References}

1 C. Risser, B. Parvitte, R. Vallon and V. Zeninari, Optimization and complete characterization of a photoacoustic gas detector, Appl. Phys. B: Lasers Opt., 2014, 118, 319-326.

2 R. E. Lindley, A. M. Parkes, K. A. Keen, E. D. McNaghten and A. J. Orr-Ewing, A sensitivity comparison of three photoacoustic cells containing a single microphone, a differential dual microphone or a cantilever pressure sensor, Appl. Phys. B: Lasers Opt., 2007, 86, 707-713.

3 F. G. C. Bijnen, J. Reuss and F. J. M. Harren, Geometrical Optimization of a Longitudinal Resonant Photoacoustic Cell for Sensitive and Fast Trace Gas Detection, Rev. Sci. Instrum., 1996, 67, 2914-2923.

4 C. Brand, A. Winkler, P. Hess, A. Miklós, Z. Bozóki and J. Sneider, Pulsed-laser excitation of acoustic modes in open high-Q photoacoustic resonators for trace gas monitoring: results for C(2)H(4), Appl. Opt., 1995, 34, 3257-3266.

5 A. A. Kosterev, F. K. Tittel, D. V. Serebryakov, A. L. Malinovsky and I. V. Morozov, Applications of quartz tuning forks in spectroscopic gas sensing, Rev. Sci. Instrum., 2005, 76, 043105.

6 A. A. Kosterev, T. S. Mosely and F. K. Tittel, Impact of humidity on quartz-enhanced photoacoustic spectroscopy based detection of HCN, Appl. Phys. B: Lasers Opt., 2006, 85, 295-300.

7 W. P. Arnott, J. W. Walker, H. Moosmüller, R. A. Elleman, H. H. Jonsson, G. Buzorius, W. C. Conant, R. C. Flagan and J. H. Seinfeld, Photoacoustic insight for aerosol light absorption aloft from meteorological aircraft and comparison with particle soot absorption photometer measurements: DOE Southern Great Plains climate research facility and the coastal stratocumulus imposed perturbation, J. Geophys. Res., 2006, 111, D05S02.

8 N. Bluvshtein, J. M. Flores, Q. He, E. Segre, L. Segev, N. Hong, A. Donohue, J. N. Hilfiker and Y. Rudich, Calibration of a multi-pass photoacoustic spectrometer cell using light-absorbing aerosols, Atmos. Meas. Tech., 2017, 10, 1203-1213.

9 E. C. Browne, X. Zhang, J. P. Franklin, K. J. Ridley, T. W. Kirchstetter, K. R. Wilson, C. D. Cappa and J. H. Kroll, Effect of heterogeneous oxidative aging on light absorption by biomass burning organic aerosol, Aerosol Sci. Technol., 2019, 53, 663-674.

10 N. W. Davies, M. I. Cotterell, C. Fox, K. Szpek, J. M. Haywood and J. M. Langridge, On the accuracy of aerosol photoacoustic spectrometer calibrations using absorption by ozone, Atmos. Meas. Tech., 2018, 11, 2313-2324.

11 M. E. Diveky, S. Roy, J. W. Cremer, G. David and R. Signorell, Assessing relative humidity dependent photoacoustics to retrieve mass accommodation coefficients of single optically trapped aerosol particles, Phys. Chem. Chem. Phys., 2019, 21, 4721-4731.

12 K. Foster, R. Pokhrel, M. Burkhart and S. Murphy, A novel approach to calibrating a photoacoustic absorption spectrometer using polydisperse absorbing aerosol, Atmos. Meas. Tech., 2019, 12, 3351-3363.

13 D. A. Lack, M. S. Richardson, D. Law, J. M. Langridge, C. D. Cappa, R. J. McLaughlin and D. M. Murphy, Aircraft Instrument for Comprehensive Characterization of Aerosol Optical Properties, Part 2: Black and Brown Carbon Absorption and Absorption Enhancement Measured with Photo Acoustic Spectroscopy, Aerosol Sci. Technol., 2012, 46, 555-568.

14 H. Moosmüller, R. K. Chakrabarty and W. P. Arnott, Aerosol light absorption and its measurement: A review, J. Quant. Spectrosc. Radiat. Transfer, 2009, 110, 844-878.

15 N. W. Davies, C. Fox, K. Szpek, M. I. Cotterell, J. W. Taylor, J. D. Allan, P. I. Williams, J. Trembath, J. M. Haywood and J. M. Langridge, Evaluating biases in filter-based aerosol absorption measurements using photoacoustic spectroscopy, Atmos. Meas. Tech., 2019, 12, 3417-3434.

16 F. Peers, P. Francis, C. Fox, S. J. Abel, K. Szpek, M. I. Cotterell, N. W. Davies, J. M. Langridge, K. G. Meyer, S. E. Platnick and J. M. Haywood, Observation of absorbing aerosols above clouds over the south-east Atlantic Ocean from the geostationary satellite SEVIRI - Part 1: Method description and sensitivity, Atmos. Chem. Phys., 2019, 19, 9595-9611.

17 F. Peers, P. Francis, S. J. Abel, P. A. Barrett, K. N. Bower, M. I. Cotterell, I. Crawford, N. W. Davies, C. Fox, S. Fox, J. M. Langridge, K. G. Meyer, S. E. Platnick, K. Szpek and J. M. Haywood, Observation of absorbing aerosols above clouds over the South-East Atlantic Ocean from the geostationary satellite SEVIRI - Part 2: Comparison with MODIS and aircraft measurements from the CLARIFY-2017 field campaign, Atmos. Chem. Phys. Discuss., 2020, 1-30, DOI: 10.5194/acp-2019-1176. 
18 J. W. Taylor, H. Wu, K. Szpek, K. Bower, I. Crawford, M. J. Flynn, P. I. Williams, J. Dorsey, J. Langridge, M. I. Cotterell, C. Fox, N. W. Davies, J. M. Haywood and $\mathrm{H}$. Coe, Absorption closure in highly aged biomass burning smoke, Atmos. Chem. Phys., 2020, 20, 11201-11221.

19 H. Wu, J. W. Taylor, K. Szpek, J. M. Langridge, P. I. Williams, M. Flynn, J. D. Allan, S. J. Abel, J. Pitt, M. I. Cotterell, C. Fox, N. W. Davies, J. M. Haywood and H. Coe, Vertical variability of the properties of highly aged biomass burning aerosol transported over the southeast Atlantic during CLARIFY2017, Atmos. Chem. Phys., 2020, 20, 12697-12719.

20 P. Stier, J. H. Seinfeld, S. Kinne and O. Boucher, Aerosol absorption and radiative forcing, Atmos. Chem. Phys., 2007, 7, 5237-5261.

21 G. Myhre, B. H. Samset, C. W. Mohr, K. Alterskjær, Y. Balkanski, N. Bellouin, M. Chin, J. Haywood, Ø. Hodnebrog, S. Kinne, G. Lin, M. T. Lund, J. E. Penner, M. Schulz, N. Schutgens, R. B. Skeie, P. Stier, T. Takemura and K. Zhang, Cloudy-sky contributions to the direct aerosol effect, Atmos. Chem. Phys., 2020, 20, 8855-8865.

22 M. I. Cotterell, K. Szpek, J. M. Haywood and J. M. Langridge, Sensitivity and accuracy of refractive index retrievals from measured extinction and absorption cross sections for mobility-selected internally mixed light absorbing aerosols, Aerosol Sci. Technol., 2020, 54(9), 1034-1057.

23 D. Al Fischer and G. D. Smith, Can ozone be used to calibrate aerosol photoacoustic spectrometers?, Atmos. Meas. Tech., 2018, 11, 6419-6427.

24 M. I. Cotterell, A. J. Orr-Ewing, K. Szpek, J. M. Haywood and J. M. Langridge, The impact of bath gas composition on the calibration of photoacoustic spectrometers with ozone at discrete visible wavelengths spanning the Chappuis band, Atmos. Meas. Tech., 2019, 12, 2371-2385.

25 A. Miklós, P. Hess and Z. Bozóki, Application of acoustic resonators in photoacoustic trace gas analysis and metrology, Rev. Sci. Instrum., 2001, 72, 1937-1955.

26 M. E. Diveky, S. Roy, G. David, J. W. Cremer and R. Signorell, Fundamental investigation of photoacoustic signal generation from single aerosol particles at varying relative humidity, Photoacoustics, 2020, 18, 100170.

27 J. W. Cremer, K. M. Thaler, C. Haisch and R. Signorell, Photoacoustics of single laser-trapped nanodroplets for the direct observation of nanofocusing in aerosol photokinetics, Nat. Commun., 2016, 7, 10941.

28 D. M. Murphy, The Effect of Water Evaporation on Photoacoustic Signals in Transition and Molecular Flow, Aerosol Sci. Technol., 2009, 43, 356-363.

29 J. Kalkman and H. W. van Kesteren, Relaxation effects and high sensitivity photoacoustic detection of NO2 with a blue laser diode, Appl. Phys. B: Photophys. Laser Chem., 2008, 90, 197-200.

30 M. I. Cotterell, G. P. Ward, A. P. Hibbins, J. M. Haywood, A. Wilson and J. M. Langridge, Optimizing the performance of aerosol photoacoustic cells using a finite element model. Part 1: Method validation and application to single-resonator multipass cells, Aerosol Sci. Technol., 2019, 53, 1107-1127.
31 M. I. Cotterell, G. P. Ward, A. P. Hibbins, A. Wilson, J. M. Haywood and J. M. Langridge, Optimizing the performance of aerosol photoacoustic cells using a finite element model. Part 2: Application to a two-resonator cell, Aerosol Sci. Technol., 2019, 53, 1128-1148.

32 D. A. Lack, E. R. Lovejoy, T. Baynard, A. Pettersson and A. R. Ravishankara, Aerosol Absorption Measurement using Photoacoustic Spectroscopy: Sensitivity, Calibration, and Uncertainty Developments, Aerosol Sci. Technol., 2006, 40, 697-708.

33 K. J. Zarzana, C. D. Cappa and M. A. Tolbert, Sensitivity of Aerosol Refractive Index Retrievals Using Optical Spectroscopy, Aerosol Sci. Technol., 2014, 48, 1133-1144.

34 R. E. H. Miles, S. Rudić, A. J. Orr-Ewing and J. P. Reid, Sources of Error and Uncertainty in the Use of Cavity Ring Down Spectroscopy to Measure Aerosol Optical Properties, Aerosol Sci. Technol., 2011, 45, 1360-1375.

35 W. P. Arnott, H. Moosmüller and J. W. Walker, Nitrogen dioxide and kerosene-flame soot calibration of photoacoustic instruments for measurement of light absorption by aerosols, Rev. Sci. Instrum., 2000, 71, 4545.

36 T. Nakayama, H. Suzuki, S. Kagamitani, Y. Ikeda, A. Uchiyama and Y. Matsumi, Characterization of a Three Wavelength Photoacoustic Soot Spectrometer (PASS-3) and a Photoacoustic Extinctiometer (PAX), J. Meteorol. Soc. Japan Ser. II, 2015, 93, 285-308.

37 D. Fischer and G. D. Smith, A portable, four-wavelength, single-cell photoacoustic spectrometer for ambient aerosol absorption, Aerosol Sci. Technol., 2018, 52, 393-406.

38 D. A. Lack, M. S. Richardson, D. Law, J. M. Langridge, C. D. Cappa, R. J. McLaughlin and D. M. Murphy, Aircraft Instrument for Comprehensive Characterization of Aerosol Optical Properties, Part 2: Black and Brown Carbon Absorption and Absorption Enhancement Measured with Photo Acoustic Spectroscopy, Aerosol Sci. Technol., 2012, 46, 555-568.

39 C. D. Cappa, T. B. Onasch, P. Massoli, D. R. Worsnop, T. S. Bates, E. S. Cross, P. Davidovits, J. Hakala, K. L. Hayden, B. T. Jobson, K. R. Kolesar, D. A. Lack, B. M. Lerner, S.-M. Li, D. Mellon, I. Nuaaman, J. S. Olfert, T. Petaja, P. K. Quinn, C. Song, R. Subramanian, E. J. Williams and R. A. Zaveri, Radiative Absorption Enhancements Due to the Mixing State of Atmospheric Black Carbon, Science, 2012, 337, 1078-1081.

40 B. Mason, N. L. Wagner, G. Adler, E. Andrews, C. A. Brock, T. D. Gordon, D. A. Lack, A. E. Perring, M. S. Richardson, J. P. Schwarz, M. A. Shook, K. L. Thornhill, L. D. Ziemba and D. M. Murphy, An intercomparison of aerosol absorption measurements conducted during the SEAC 4 RS campaign, Aerosol Sci. Technol., 2018, 52, 1012-1027.

41 J. B. Burkholder, S. P. Sander, J. P. D. Abbatt, J. R. Barker, R. E. Huie, C. E. Kolb, M. J. Kurylo, V. L. Orkin, D. M. Wilmouth and P. H. Wine, Chemical Kinetics and Photochemical Data for Use in Atmospheric Studies, Evaluation Number 18, JPL Publ, 2015.

42 N. Sharma, I. J. Arnold, H. Moosmüller, W. P. Arnott and C. Mazzoleni, Photoacoustic and nephelometric spectroscopy 
of aerosol optical properties with a supercontinuum light source, Atmos. Meas. Tech., 2013, 6, 3501-3513.

43 S. Y. Grebenshchikov, Z. W. Qu, H. Zhu and R. Schinke, Phys. Chem. Chem. Phys., 2007, 9, 2044-2064.

44 R. Kaiser, On the theory of the spectrophone, Can. J. Phys., 1959, 37, 1499-1513.

45 H. Flöthmann, R. Schinke, C. Woywod and W. Domcke, Photodissociation of ozone in the Chappuis band. III. Product state distributions, J. Chem. Phys., 1998, 109, 2680-2684.

46 H. B. Levene, J. Nieh and J. J. Valentini, Ozone visible photodissociation dynamics, J. Chem. Phys., 1987, 87, 2583-2593.

47 W. G. Thomas, M. J. Preslar and J. C. Farmer, Calibration of Condenser Microphones under Increased Atmospheric Pressures, J. Acoust. Soc. Am., 1972, 51, 6-14.

48 C. H. Chan, Effective absorption for thermal blooming due to aerosols, Appl. Phys. Lett., 1975, 26, 628-630.

49 Y.-H. Pao, Optoacoustic Spectroscopy and Detection, Academic Press, New York, 1st edn, 1977.

50 A. Rosencwaig, Photoacoustics and Photoacoustic Spectroscopy, John Wiley and Sons, Inc., 1980.

51 C. W. Tan, Z. Wang, J. Miao and X. Chen, A study on the viscous damping effect for diaphragm-based acoustic MEMS applications, J. Micromech. Microeng., 2007, 17, 2253-2263.

52 P. A. Tipler and G. Mosca, Physics for Scientists and Engineers, W. H. Freeman and Company, New York, 6th edn, 2008.
53 F. Ménard-Bourcin, J. Ménard and L. Doyennette, Vibrational relaxation of ozone in $\mathrm{O} 3-\mathrm{O} 2$ and $\mathrm{O} 3-\mathrm{N} 2$ gas mixtures from infrared double-resonance measurements, J. Chem. Phys., 1991, 94, 1875-1881.

54 V. Zeninari, B. A. Tikhomirov, Y. N. Ponomarev and D. Courtois, Photoacoustic measurements of the vibrational relaxation of the selectively excited ozone (v3) molecule in pure ozone and its binary mixtures with O2, N2, and noble gases, J. Chem. Phys., 2000, 112, 1835-1843.

55 R. Siebert, P. Fleurat-Lessard, R. Schinke, M. Bittererová and S. C. Farantos, The vibrational energies of ozone up to the dissociation threshold: Dynamics calculations on an accurate potential energy surface, J. Chem. Phys., 2002, 116, 9749-9767.

56 C. Coletti and G. D. Billing, Vibrational energy transfer in molecular oxygen collisions, Chem. Phys. Lett., 2002, 356, 14-22.

57 D. L. Huestis, Vibrational Energy Transfer and Relaxation in $\mathrm{O} 2$ and H2O, J. Phys. Chem. A, 2006, 110, 6638-6642.

58 H. Park and T. G. Slanger, O2 (X, v = 8-22) $300 \mathrm{~K}$ quenching rate coefficients for $\mathrm{O} 2$ and $\mathrm{N} 2$, and $\mathrm{O} 2(\mathrm{x})$ vibrational distribution from $248 \mathrm{~nm}$ O3 photodissociation, J. Chem. Phys., 1994, 100, 287-300.

59 K. M. Hickson, P. Sharkey, I. W. M. Smith, A. C. Symonds, R. P. Tuckett and G. N. Ward, Formation and relaxation of $\mathrm{O} 2(\mathrm{X} 3 \Sigma \mathrm{g}-)$ in high vibrational levels $(18<\mathrm{v}<23)$ in the photolysis of $\mathrm{O} 3$ at $266 \mathrm{~nm}, \mathrm{~J}$. Chem. Soc., Faraday Trans., 1998, 94, 533-540.

60 G. D. Billing, VV and VT rates in N2-O2 collisions, Chem. Phys., 1994, 179, 463-467. 